\title{
WestVirginiaUniversity
}

THE RESEARCH REPOSITORY @ WVU

Graduate Theses, Dissertations, and Problem Reports

2013

\section{Circuits, Perfect Matchings and Paths in Graphs}

Wenliang Tang

West Virginia University

Follow this and additional works at: https://researchrepository.wvu.edu/etd

\section{Recommended Citation}

Tang, Wenliang, "Circuits, Perfect Matchings and Paths in Graphs" (2013). Graduate Theses, Dissertations, and Problem Reports. 386.

https://researchrepository.wvu.edu/etd/386

This Dissertation is protected by copyright and/or related rights. It has been brought to you by the The Research Repository @ WVU with permission from the rights-holder(s). You are free to use this Dissertation in any way that is permitted by the copyright and related rights legislation that applies to your use. For other uses you must obtain permission from the rights-holder(s) directly, unless additional rights are indicated by a Creative Commons license in the record and/ or on the work itself. This Dissertation has been accepted for inclusion in WVU Graduate Theses, Dissertations, and Problem Reports collection by an authorized administrator of The Research Repository @ WVU.

For more information, please contact researchrepository@mail.wvu.edu. 


\title{
Circuits, Perfect Matchings and Paths in Graphs
}

\author{
Wenliang Tang \\ Dissertation submitted to the \\ Eberly College of Arts and Sciences \\ West Virginia University \\ in partial fulfillment of the requirements \\ for the degree of \\ Doctor of Philosophy \\ in \\ Mathematics \\ Cun-Quan Zhang, Ph.D., Chair \\ Mark Culp, Ph.D. \\ John Goldwasser, Ph.D. \\ Hong-Jian Lai, Ph.D. \\ Jerzy Wojciechowski, Ph.D. \\ Department of Mathematics \\ Morgantown, West Virginia \\ 2013
}

Keywords: Strong Circuit Double Cover Conjecture, Berge-Fulkerson Conjecture, Saturation Number, Turan Number

Copyright 2013 Wenliang Tang 


\section{ABSTRACT \\ Circuits, Perfect Matchings and Paths in Graphs}

\section{Wenliang Tang}

We primarily consider the problem of finding a family of circuits to cover a bidgeless graph (mainly on cubic graph) with respect to a given weight function defined on the edge set. The first chapter of this thesis is going to cover all basic concepts and notations will be used and a survey of this topic.

In Chapter two, we shall pay our attention to the Strong Circuit Double Cover Conjecture (SCDC Conjecture). This conjecture was verified for some graphs with special structure. As the complement of two factor in cubic graph, the Berge-Fulkersen Conjecture was introduced right after SCDC Conjecture. In Chapter three, we shall present a series of conjectures related to perfect matching covering and point out their relationship.

In last chapter, we shall introduce the saturation number, in contrast to extremal number (or known as Turán Number), and describe the edge spectrum of saturation number for small paths, where the spectrum was consisted of all possible integers between saturation number and Turán number. 


\section{Acknowledgements}

First and foremost, I am most indebted to my supervisor, Dr. Cun-Quan Zhang, for his continued encouragement and support over these last few years. It is a pleasure to work under his supervision. Without him, this paper could not have come about.

I would also like to thank my other committee members: Dr. Mark Culp, Dr. John Goldwasser, Dr. Hong-Jian Lai and Dr. Jerzy Wojciechowski, for their help during my studies.

And finally, I would like to thank the Department of Mathematics and Eberly College of Arts and Sciences at West Virginia University for providing me with an excellent study environment and support during my study as a graduate student. 


\section{Contents}

1 Circuit Cover 1

1.1 Notation and Terminology . . . . . . . . . . . . . . . . . . 1

1.2 Circuit Double Cover . . . . . . . . . . . . . . . . . . 2

1.3 Faithful Circuit Cover . . . . . . . . . . . . . . . . . 3

1.4 Strong Circuit Double Cover . . . . . . . . . . . . . . . . . . 5

2 Strong Circuit Double Cover Conjecture $\quad 6$

2.1 Introduction . . . . . . . . . . . . . . . . . 6

2.2 Lollipop method and its applications . . . . . . . . . . . . . 9

$2.3 G-V(C)$ has a Hamilton path or $Y$-tree $\ldots \ldots \ldots$. . . . . . . . 11

$2.4 H=G-V(C)$ is connected . . . . . . . . . . . . . . 14

2.5 Girth requirement for counterexample to SCDC . . . . . . . . . . . 16

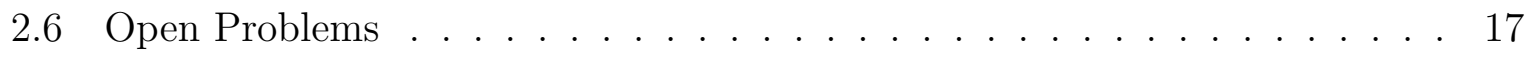

3 Berge-Fulkerson Conjecture $\quad 19$

3.1 Introduction . . . . . . . . . . . . . . . . . . . . . 19

3.2 Perfect matching covering for weighted graphs . . . . . . . . . . . 22

3.2.1 Fan-Raspaud Conjecture and matching coverage conjectures . . . . 23 
$3.33 P M$-Coverage Index - a rank for snarks . . . . . . . . . . . . . 26

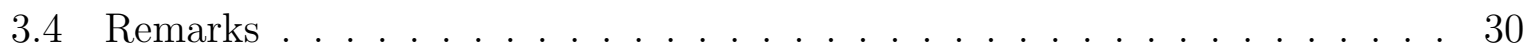

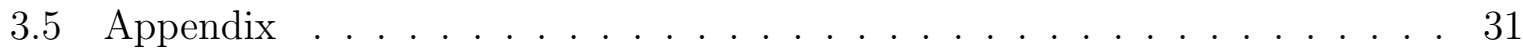

3.5.1 The perfect matching polytope ............... 31

4 Saturation Number for Paths $\quad 35$

4.1 Introduction and Notation . . . . . . . . . . . . . . . 35

4.2 Known Results about Extremal Number on Paths . . . . . . . . . . . . . . 36

4.3 Edge Spectrum of $P_{5}$-Saturated Graphs . . . . . . . . . . . . . . . 38

4.4 Edge Spectrum of $P_{6}$-Saturated Graphs . . . . . . . . . . . . . . . . 42 


\section{Chapter 1}

\section{Circuit Cover}

\section{$1.1 \quad$ Notation and Terminology}

We use [6] for terminology and notations not defined here. Graphs in this dissertation are finite and may have multiple edges but no loops. Let $G$ be a graph. We use $V(G)$ and $E(G)$ to denote the set of vertices and the set of edges of $G$, respectively. Two vertices $u, v$ are adjacent if $u v \in E(G)$.

For a graph $G$ and for $v \in V(G)$, the neighborhood $N_{G}(v)$ denotes the set of all vertices adjacent to $v$ in $G$. The cardinality of $N_{G}(v)$ is called the degree of $v$ in $G$, and is denoted by $d_{G}(v)$ or $d(v)$. For a vertex subset $A$ of $G$.

Let $X \subseteq E(G)$. The contraction $G / X$ is the graph obtained from $G$ by identifying the two ends of each edge in $X$ and then deleting the resulting loops. If $H$ is a subgraph of $G$, we write $G / H$ for $G / E(H)$. Note that even if $G$ is a simple graph, contracting some edges of $G$ may result in a graph with multiple edges.

Let $G=(V, E)$ be a graph with vertex set $V$ and edge set $E$. A circuit is a connected 2-regular graph. A graph (subgraph) is even(also called cycle sometimes in this paper) if the degree of each vertex is even. A bridge (or, cut-edge) of a graph $G$ is an edge whose removal increases the number of components of $G$ (equivalently, a bridge is an edge that is not contained in any circuit of $G$ ). 


\subsection{Circuit Double Cover}

Definition 1.2.1 Let $\mathcal{F}$ be a family of circuits (or, cycles) of a graph $G$.

1. $\mathcal{F}$ is called a circuit cover (or a cycle cover) if every edge of $G$ is contained in some members of $\mathcal{F}$.

2. A circuit cover (or, a cycle cover) $\mathcal{F}$ of a graph $G$ is called a double cover of $G$ if every edge is contained in precisely two members of $\mathcal{F}$.

The following is one of the most well-known open problems in graph theory.

Conjecture 1.2.2 (Circuit Double Cover Conjecture) Every bridgeless graph G has a family $\mathcal{F}$ of circuits that every edge of $G$ is contained in precisely two members of $\mathcal{F}$.

Figure 1.1 shows an example of circuit covers of $K_{4}$.
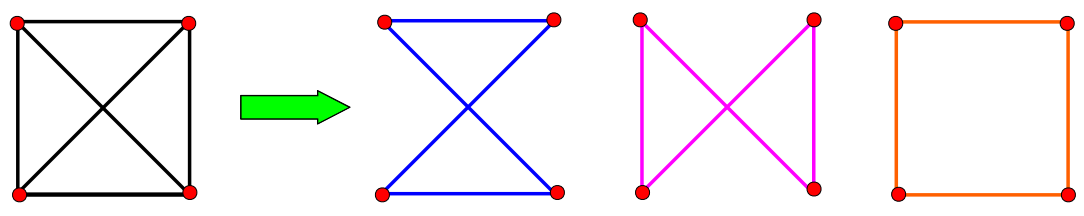

Figure 1.1: A circuit double cover of $K_{4}$.

The circuit double cover (CDC) conjecture is obviously true for 2-connected planar graphs by taking the set of the boundaries of all faces. The conjecture also holds for the family of 3-edge-colorable cubic graphs since any three-edge-colorable cubic graph is covered by the family of bi-colored circuits. The same observation will be further extended to all bridgeless graphs (not necessary cubic) admitting nowhere-zero 4-flows.

We would like to establish some properties of bridge less graphs which have no circuit double covers. In studying such counterexamples to the CDC conjecture, one gains insight toward the natural of the conjecture.

With some straightforward observations, the following theorem summarizes some structure of a minimal counterexample to the CDC conjecture. 
A standard technique involves taking a smallest counterexample $G$, one which has a minimum number of edges. From $G$ we can obtain a smaller bridgeless graph by deleting an edge, or by some operation such as contraction. By the choice of $G$, the smaller new graph has a double cover. We then attempt to modify the family of circuits in order to obtain a circuit double cover of $G$.

Theorem 1.2.3 Let $G$ be a minimal counterexample to $C D C$ conjecture, then

1. G is simple, 3-connected and cubic;

2. G has no nontrivial 2 or 3-edge-cut;

3. $G$ is not 3-edge-colorable;

4. $G$ is not planar;

There are more structural properties about the minimal counterexample to CDC conjecture. The detail proofs of those properties can be found in [66].

\subsection{Faithful Circuit Cover}

The concept of faithful circuit cover is not only a generalization of the circuit double cover, but also an inductive approach to the CDC conjecture in a very natural way. Let $\mathbb{Z}^{+}$be the set of all positive integers, and $\mathbb{Z}^{*}$ be the set of all non-negative integers.

Definition 1.3.1 Let $G$ be a graph and $\omega: E(G) \mapsto \mathbb{Z}^{+}$. A family $\mathcal{F}$ of circuits (or, cycles) of a graph $G$ is a faithful circuit (or, cycle) cover with respect to $\omega$ if each edge $e$ of $G$ is contained in precisely $\omega(e)$ members of $\mathcal{F}$.

Figure 1.2 shows an example of faithful circuit covers of $\left(K_{4}, \omega\right)$, where $\omega$ assigns 2 to the pair of diagonals and 1 to 4 sides.

It is obviously that the CDC conjecture is a special case of the faithful circuit cover problem with $\omega(e)=2$ for each edge of $G$. 


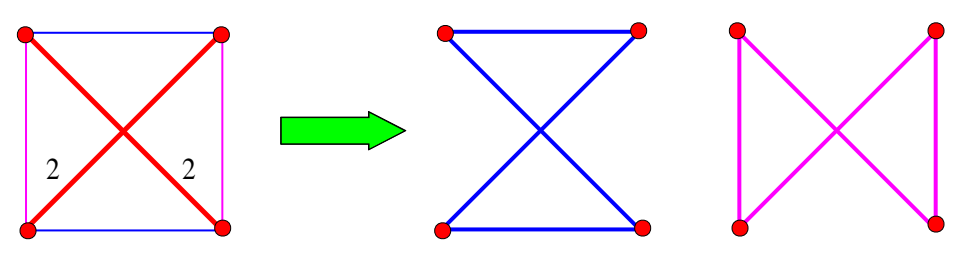

Figure 1.2: A faithful circuit cover of $K_{4}$ with respect to $\omega$.

Definition 1.3.2 Let $G$ be a graph and $\omega: E(G) \mapsto \mathbb{Z}^{+}$be a weight. Then,

1. $(G, \omega)$ is called an Eulerian weighted graph if $\omega$ is eulerian, i.e. the total weight of every edge-cut of $G$ is even;

2. An eulerian weight $\omega$ is called admissible if, for every edge-cut $T$ and every $e \in E(T)$,

$$
\omega(e) \leq \frac{\omega(T)}{2}
$$

The requirements of being eulerian and admissible are necessary for faithful circuit covers based on the observation that each circuit crosses very edge-cut with even times. A question would be asked naturally as follows.

Question 1.3.3 Let $G$ be a graph and $\omega: E(G) \mapsto \mathbb{Z}^{+}$be an admissible and eulerian weight. Does $G$ have a faithful circuit cover with respect to $\omega$ ?

Unfortunately, the answer is NOT always true. The Petersen graph $P_{10}$ with an eulerian weight $\omega_{10}$, assigning 2 to the perfect matching connecting two pentagons, does not have a faithful circuit cover with respect to $\omega_{10}$ (See Figure 1.3).

Definition 1.3.4 Let $G$ be a bridge less graph and $\omega$ be an admissible eulerian weight of $G$. The eulerian weighted graph $(G, \omega)$ is a contra pair if $G$ does not have a faithful circuit cover with respect to the weight $\omega$.

For a given weight $\omega: E(G) \mapsto \mathbb{Z}^{+}$, denote $E_{\omega=i}=\{e \in E(G): \omega(e)=i\}$. An admissible pair $(G, \omega)$ is a contra pair if it has no faithful circuit cover, and a contra pair 


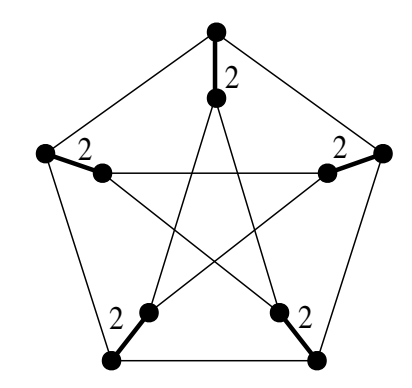

Figure 1.3: A contra pair $\left(P_{10}, \omega_{10}\right)$

is minimal if $G$ is cubic and $(G, \omega)$ has no removable circuit, but, for every edge $e$ with weight 2 , the graph $G-\{e\}$ has a faithful circuit cover with respect to $\omega$.

It is proved by Alspach et al. [1] that if $(G, \omega)$ is a minimal contra pair, then the graph $G$ must contain a Petersen minor. It is further conjectured by Fleischner and Jackson ([20], also see [31]) that this graph $G$ must be Petersen graph itself (not just as a minor).

\subsection{Strong Circuit Double Cover}

One may note in above figure that $E_{\omega=1}$ induces two disjoint circuits in Petersen graph. What if for an eulerian $(1,2)$-weighted graph $(G, \omega)$ the induced graph $E_{\omega=1}$ is a single circuit? The next conjecture is the main problem we paid much attention.

Conjecture 1.4.1 (Strong Circuit Double Cover (SCDC) Conjecture, Seymour, see [21] p. 237, and [23], also see [29] ) Let $\omega: E(G) \mapsto \mathbb{Z}^{+}$be an admissible and eulerian weight of a bridge less graph $G$. If the subgraph induced by the weight one edges is a circuit, then $(G, \omega)$ has a faithful circuit cover $\mathcal{F}$.

The above conjecture can also be presented in terms of circuit as follows.

Conjecture 1.4.2 Strong Circuit Double Cover Conjecture Let $C$ be a circuit of a bridgeless cubic graph $G$. Then there exists a family $\mathcal{F}$ of circuits of $G$ covers every edge of $G$ twice and contains $C$ also. $\mathcal{F}$ is called a strong circuit double cover of $G$ with respect to $C$. 


\section{Chapter 2}

\section{Strong Circuit Double Cover Conjecture}

\section{$2.1 \quad$ Introduction}

The strong circuit double cover conjecture has been recognized as one of the major open problems in graph theory. Let's restate it to start this chapter:

Let $G$ be a bridgeless cubic graph and $C$ be any given circuit in $G$, then the graph $G$ has a circuit double cover $\mathcal{F}$ containing $C$.

The SCDC conjecture has been verified for various families of graphs, such as, 3-edgecolorable cubic graphs [54], snarks of order at most 36 [9], a circuit $C$ of length at least $|V(G)|-1$ [26], and some special families of graphs with given circuits described in [24], [37] (see Theorems 2.1.2, 2.1.5), etc.

Note that the SCDC Conjecture is not true if the given circuit $C$ is replaced with a family of edge-disjoint circuits (the Petersen graph is a counterexample).

The next conjecture has been verified by Tarsi for graphs with Hamilton paths.

Theorem 2.1.1 (Tarsi [57]) Every bridgeless cubic graph containing a Hamilton path has a circuit double cover. 
Theorem 2.1.1 is further strengthened in [37] with respect to Conjecture 1.4.2 (the SCDC Conjecture).

Theorem 2.1.2 (Fleischner and Häggkvist[37]) Let $G$ be a bridgeless cubic graph with a Hamilton path $v_{1} \ldots v_{n}$ and $v_{1} v_{h} \in E(G)(h>2)$. Then $G$ has a $C D C \mathcal{F}$ that contains the circuit $v_{1} \ldots v_{h} v_{1}$.

In this chapter, we are interested in extending Theorems 2.1.1 and 2.1.2 as follows.

Problem 2.1.3 Let $G$ be a bridgeless cubic graph with a given circuit $C$. If $G-V(C)$ contains a Hamilton path $P$, can we find a $C D C \mathcal{F}$ of $G$ that contains the circuit $C$ ?

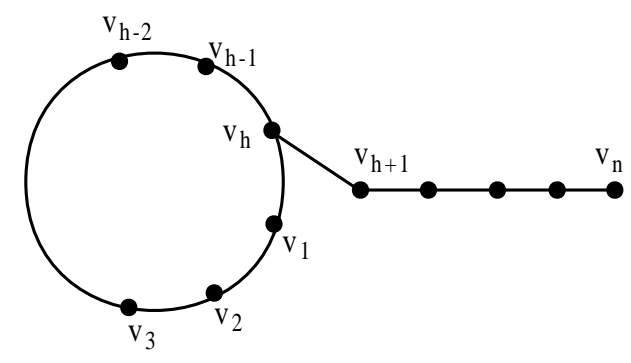

(a) Theorems 2.1.2

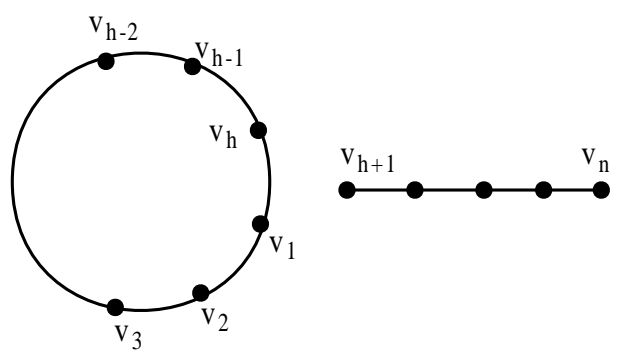

(b) Problem 2.1.3

Figure 2.1: A Hamilton path $v_{1} v_{2} \cdots v_{h} v_{h+1} \cdots v_{n}$ can be found on left figure. There is no Hamilton path on right figure, i.e. two end vertices $v_{h+1}$ and $v_{n}$ are not adjacent to the circuit $v_{1} v_{2} \cdots v_{h}$.

Or, a more general question as following.

Problem 2.1.4 Let $G$ be a bridgeless cubic graph with a given circuit $C$. If $G-V(C)$ is connected, can we find a $C D C \mathcal{F}$ of $G$ that contains the circuit $C$ ?

For Problem 2.1.4, Fleischner and Häggkvist has the following partial result. 
Theorem 2.1.5 (Fleischner and Häggkvist [24]) Let $G$ be a bridgeless cubic graph with a given circuit $C$. If $G-V(C)$ is connected and of order at most 4 , then $G$ has a $C D C \mathcal{F}$ that contains the circuit $C$.

Note that the difference between Theorem 2.1.2 and Problem 2.1.3 is whether there is an edge joining an endvertex of $P$ and some vertex of $C$. If yes, the lollipop method (Section 2.2) is applied and Theorem 2.1.2 follows [37]. However, if the circuit $C$ and the path $P$ are not connected in such way, more structural studies are necessary beyond the application of the lollipop method (see Figure 2.1).

In this chapter, we obtained some partial results (Theorems 2.1.8, 2.1.9) related to both problems that strengthen some of those results by Fleischner and Häggkvist.

Almost all results in this paper are presented for cubic graphs only. However, they all can be converted to results for general graphs by applying vertex splitting methods [19].

For the sake of convenience, we denote $(G, C)$ a pair of a cubic graph $G$ and a given circuit $C$ of $G$.

Definition 2.1.6 Let $G$ be a graph. The suppressed graph of $G$ is the graph obtained from $G$ by replacing each maximal subdivided edge with a single edge, and is denoted by $\bar{G}$.

Definition 2.1.7 A spanning tree $T$ of the graph $H$ is called a spanning $Y$-tree if $T$ consists of a path $v_{1}, \cdots, v_{t-1}$ and $v_{t-2} v_{t} \in E(T)$. (See Figure 3.2.1.)

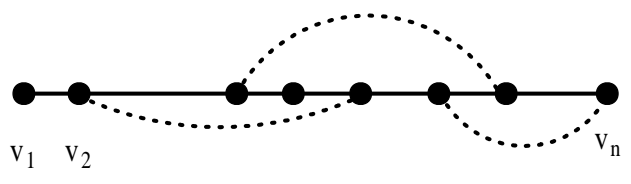

(a) Hamilton Path

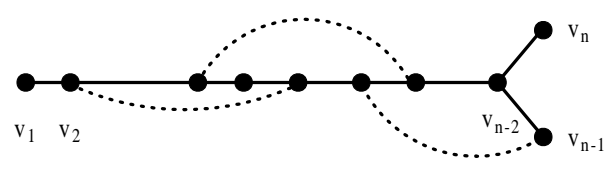

(b) Y-tree

Figure 2.2: Hamilton Path and Y-tree (solid line) spanning in $H$.

The following are main results of this chapter. 
Theorem 2.1.8 Let $C$ be a given circuit of a bridgeless cubic graph $G$. If $H=G-C$ contains a Hamilton path or a $Y$-tree of order $\leq 20$, then $G$ has a circuit double cover containing $C$.

Theorem 2.1.9 Let $C$ be a given circuit of a bridgeless cubic graph $G$. If $H=G-C$ is connected and of order $\leq 6$, then $G$ has a circuit double cover containing $C$.

\subsection{Lollipop method and its applications}

Definition 2.2.1 Let $P=v_{1} v_{2} \cdots v_{t}$ be a path of a cubic graph. Let $v_{i} \in N\left(v_{t}\right) \cap$ $\left\{v_{2}, v_{3}, \cdots, v_{t-1}\right\}$. The subgraph $P^{\prime}=v_{1} v_{2}(P) v_{i} v_{t}(P) v_{i+1}$ is a path obtained from $P$ via a lollipop detour (see Figure 2.3).
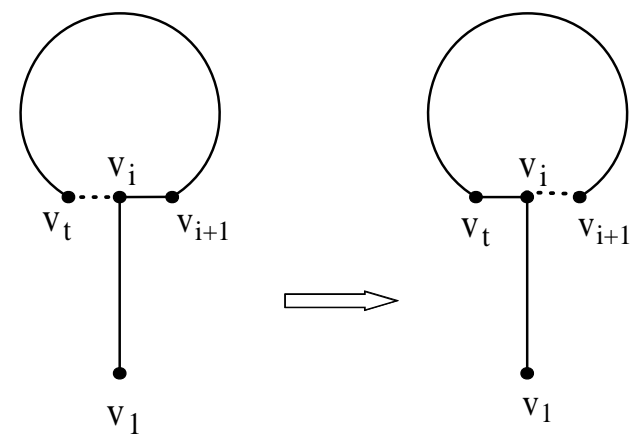

Figure 2.3: Lollipop Detour $P \Rightarrow P^{\prime}$

The following lemma shall be proved by the technique of lollipop method, which was first introduced by Thomason [58].

Lemma 2.2.2 Let $G$ be a cubic graph of order $n$ and $C=v_{1} v_{2} \cdots, v_{r} v_{1}$ be a circuit of G. Then

(1) either there is another circuit $C^{\prime}=v_{1} v_{2} \cdots v_{1}$ containing the edge $v_{1} v_{2}$ with $V(C)=V\left(C^{\prime}\right)$ and $E(C) \neq E\left(C^{\prime}\right)$;

(2) or there is a path $P=v_{1} v_{2} \cdots z$ starting at the vertex $v_{1}$ and the edge $v_{1} v_{2}$, and $V(P)=V(C) \cup z$ for some vertex $z \in V(C)$. 
Proof. Construct an auxiliary graph $A_{G}$. Each vertex of $A_{G}$ is a path $P$ of $G$ starting at the vertex $v_{1}$ and the edge $v_{1} v_{2}$ with $V(P)=V(C)$. And $P_{1}$ is connected to $P_{2}$ if and only if $P_{1}$ is obtained from $P_{2}$ via a lollipop detour. Therefore every vertex in $A_{G}$ has degree 2 or 1 .

Note that $P=v_{1} v_{2} \cdots v_{r}$ is a degree one vertex in the auxiliary graph $A_{G}$. Since the component of $A_{G}$ containing the vertex $P$ is a path and must have another degree one vertex $P^{\prime}=v_{1} v_{2} \cdots x$. The case $v_{1} \in N(x)$ implies that $P^{\prime}$ can be extended to a distinct circuit $C^{\prime}$, and otherwise $N(x)$ contains a new vertex $z$ not in $V(C)$, as we desired.

Definition 2.2.3 A Y-tree $v_{1} \cdots v_{t-1}+v_{t-2} v_{t}$ and a Hamilton path $v_{1} \cdots v_{t}$ of $H$ is small end if $d_{H}\left(v_{1}\right) \leq 2$.

In Figure 2.4, a small end Hamilton path and a small end spanning $Y$-tree are illustrated in $G-V(C)$.

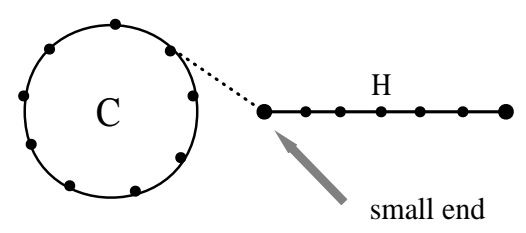

(a) Hamilton Path

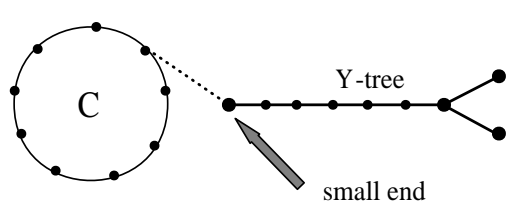

(b) Y-tree

Figure 2.4: Small end Hamilton path and small end $Y$-tree.

Here, Theorem 2.1.2 is extended as follows, which not only includes the proof of Theorem 2.1.2 but also a result for small end $Y$-trees.

Theorem 2.2.4 For a pair $(G, C)$, if $H=G-V(C)$ has either a small end Hamilton path $P_{0}=x_{1} \cdots x_{t}$ with $d_{H}\left(x_{1}\right) \leq 2$, or a small end $Y$-tree consisting of a path $x_{1} \cdots x_{t-1}$ and an edge $x_{t-2} x_{t}$, then the pair $(G, C)$ has a $C D C$ containing the circuit $C$.

Proof. Induction on $|V(G)|$. Let $C=v_{1} v_{2} \cdots v_{r} v_{1}$ be the given circuit and $T$ be the small end Hamilton path or small end spanning $Y$-tree with an end-vertex $x_{1}$ that $x_{1} v_{1} \in E(G)$. By the Lemma 2.2.2, either $G$ has a circuit $C^{\prime}$ with $V(C)=V\left(C^{\prime}\right)$ and $E(C) \neq E\left(C^{\prime}\right)$ 
or $G$ has a path $P=v_{1} v_{2} \cdots v_{j} x_{h}$ with $V(P)=V(C) \cup x_{h}$ for some vertex $x_{h}$ of $T$. The path $P$ extends $C$ to a longer circuit $C^{\prime}=v_{1} v_{2} \cdots v_{j} x_{h} T x_{1} v_{1}$.

Let $G^{\prime}=\overline{G-\left(E(C)-E\left(C^{\prime}\right)\right)}$. In either case, the reduced pair $\left(G^{\prime}, C^{\prime}\right)$ inherits the same property from $(G, C)$ : $G^{\prime}-V\left(C^{\prime}\right)$ has either a small end Hamilton path or a small end spanning $Y$-tree $T-V(P)$.

By applying induction, let $\mathcal{F}^{\prime}$ be a circuit double cover of the suppressed graph $G^{\prime}$ with $C^{\prime} \in \mathcal{F}^{\prime}$. Hence, $\mathcal{F}=\mathcal{F}^{\prime}-C^{\prime}+\left\{C^{\prime} \Delta C, C\right\}$ is a strong circuit double cover containing the circuit $C$.

\section{3 $G-V(C)$ has a Hamilton path or $Y$-tree}

Note that if $G-V(C)$ contains a Hamilton path $P$, then either $P$ is small end or $G-V(C)$ contains a circuit (since each endvertex of $P$ must adjacent to some other vertex in $P$ ).

Definition 2.3.1 Let $g_{2}$ be a largest integer such that, for every pair $(G, C)$ and for some edge e contained in a circuit $D$ of $G-V(C)$ of length less than $g_{2}$, the fact that $G-e$ has a $C D C$ containing $C$ implies that $G$ has a $C D C$ containing $C$.

In Section 2.5, we will estimate the value $g_{2}$ ( $g_{2} \geq 9$ in Lemma 2.5.1).

Theorem 2.3.2 For a pair $(G, C)$, if $H=G-V(C)$ contains a Hamilton path and is of order less than 3k-3, where $k=g_{2}(G) \geq 6$, then $G$ has a $C D C$ containing the circuit $C$.

Proof. Suppose that $(G, C)$ is counterexample to the theorem with $|V(H)|$ as small as possible.

Claim 1. We claim that every Hamilton path in $H$ has no small-end.

If there exists a small end Hamilton path $P$ in $H$, then the trheorem is true by Theorem 2.2.4 and $(G, C)$ is not a counterexample.

Since a Hamilton path $P$ is acyclic, every circuit of $H$ contains a chord of $P$. Furthermore, by deleting a chord $e$ of $P$, the pair $(\overline{G-e}, C)$ remains satisfying the theorem 
but smaller. Thus, $(G-e, C)$ has a CDC containing $C$. By the definition of $g_{2}$, we have the following conclusion.

Claim 2. The girth of $H=G-V(C)$ is at least $g_{2}$.

Claim 2 will be used frequently in the remaining part of the Proof.

Let $P=x_{1} x_{2} \cdots x_{i} \cdots x_{s} \cdots x_{t}$ be any Hamilton path in $H$ with $N\left(x_{1}\right)=\left\{x_{2}, x_{i}, x_{s}\right\}$ and $2<i<s \leq t<3 k-3$. If $s=t$, then $H$ contains a Hamilton circuit and any vertex on the circuit having a neighbor in $C$ is a small end of some Hamilton path. Thus assume that $s<t$. We choose such a Hamilton path that $s$ is maximized. By Claim 1, all neighbors of $x_{s-1}$ are contained in $P$. Furthermore, by the maximality of the integer $s, N\left(x_{s-1}\right)=\left\{x_{j}, x_{s-2}, x_{s}\right\}$, where $j<s-2$. (See Figure 2.5.)

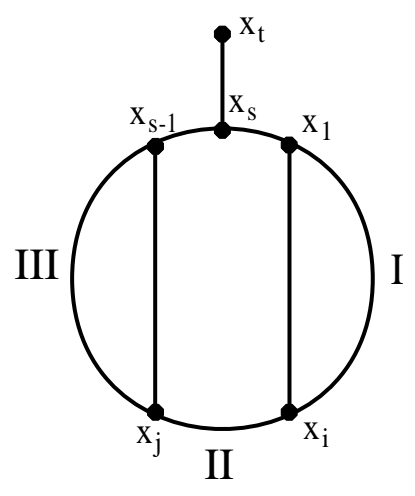

(a) Case 1: $i<j$

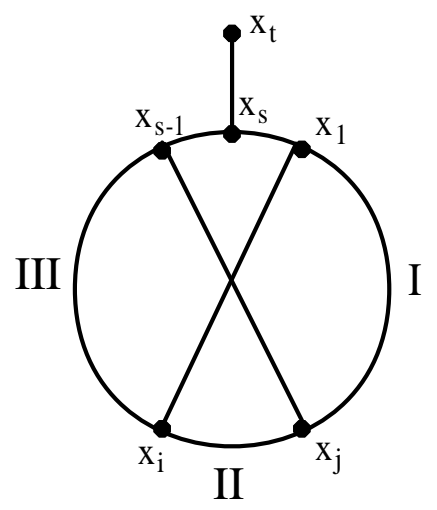

(b) Case 2: $i>j$

Figure 2.5: The local structure of $H$.

Notation: Let $p<q$ be two positive integers, denote by $|(p, q)|$ the number of integers contained in this open interval. For example, $|(3,5)|=1$.

Case 1: $i<j$. By the Definition of $g_{2}\left(g_{2}=k\right)$, we know that $|(1, i)| \geq k-2,|(i, j)| \geq$ $k-5$ and $|(j, s-1)| \geq k-2$. Therefore, $s \geq(k-2)+(k-5)+(k-2)+5=3 k-4$ and $t \geq s+1 \geq 3 k-3$. 
Case 2: $i>j$. The fact that $|(j, i)| \geq k-5 \geq 1$ comes from the circuit $x_{j} \cdots x_{i} x_{1} x_{s} x_{s-1} x_{j}$. The Hamilton path $x_{j+1} \cdots x_{s-1} x_{j} \cdots x_{1} x_{s} \cdots x_{t}$ implies that $N\left(x_{j+1}\right) \subset\left\{x_{1}, \cdots, x_{s}\right\}$ by the maximality of $s$, and denote $x_{p}=N\left(x_{j+1}\right) \backslash\left\{x_{j}, x_{j+2}\right\}$. (See Figure 2.6.)

Case 2(a): $x_{p} \in\left\{x_{2}, \cdots, x_{j-1}\right\}$. The circuit $x_{1} \cdots x_{s}$ and two chords $x_{1} x_{i}, x_{j+1} x_{p}$ imply that $s \geq 3 k-4$ and $t \geq 3 k-3$.

Case 2(b): $x_{p} \in\left\{x_{j+2}, \cdots, x_{s-2}\right\}$. The circuit $x_{1} \cdots x_{s}$ and two chords $x_{j} x_{s-1}, x_{j+1} x_{p}$ imply that $s \geq 3 k-4$ and $t \geq 3 k-3$.

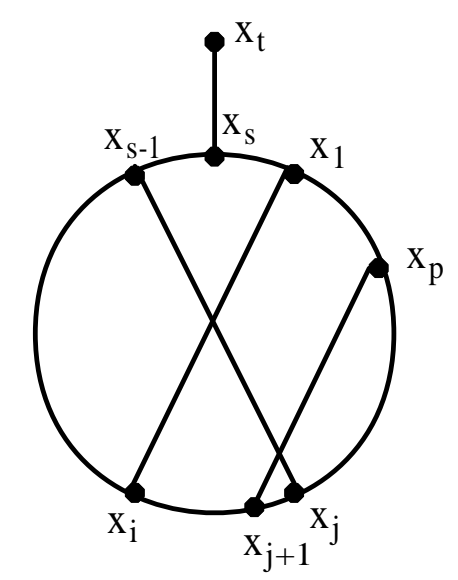

(a) $1<p<j$

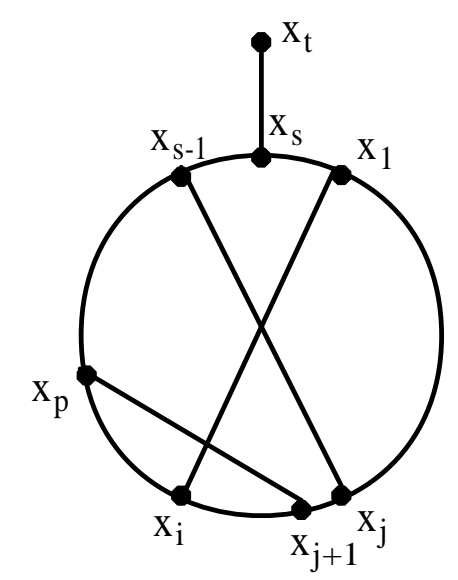

(b) $j+2<p<s-1$

Figure 2.6: The local structure of $H$.

No matter in which case, a contradiction that $t \geq 3 k-3$ is obtained.

Immediately, we get the next corollary by Lemma 2.5.1.

Corollary 2.3.3 For a pair $(G, C)$, if $H=G-V(C)$ contains a hamilton path and is of order less than 24, then $(G, C)$ has a $C D C$ containing the circuit $C$.

Theorem 2.3.4 For a pair $(G, C)$, if $H=G-V(C)$ contains a spanning $Y$-tree and is of order less than $3 k-3$, where $k=g_{2}(G) \geq 6$, then $(G, C)$ has a $C D C$ containing the circuit $C$. 
Proof. It can be proved by Theorem 2.2.4 if there exists a small end spanning $Y$-tree in $H$. Thus we may assume that any spanning $Y$-tree in $H$ has no small end vertex.

Let $Y=x_{1} x_{2} \cdots x_{t-2} x_{t-1}+x_{t-1} x_{t}$ be any spanning $Y$-tree with $N\left(x_{1}\right)=\left\{x_{2}, x_{i}, x_{s}\right\}$ with $2<i<s \leq t<3 k-3$. If $s \in\{t-1, t\}$, then $G-V(C)$ has a Hamilton path and is proved by Theorem 2.3.2. So assume that $s<t-2$. We can choose such a $Y$-tree that $s$ is maximized.

With a similar Proof of Theorem 2.3.2 (detail omitted), we can prove that

$$
s \geq 3 k-4,
$$

which implies that $t \geq(3 k-4)+3=3 k-1$ and contradicts the fact $t<3 k-3$.

Corollary 2.3.5 For a pair $(G, C)$, if $H=G-V(C)$ contains a spanning $Y$-tree and is of order less than 24 , then $(G, C)$ has a $C D C$ containing the circuit $C$.

The combination of Corollaries 2.3.3 and 2.3.5 yields Theorem 2.1.8

The next corollary can be derived straightly from the above two theorems, and slightly improves an early result by Fleischner and Häggkvist [24] for $|V(H)| \leq 4$ and $H$ is connected.

Corollary 2.3.6 For a pair $(G, C)$, if $H=G-V(C)$ is connected and of order at most 5 , then $(G, C)$ has a $C D C$ containing the circuit $C$.

Proof. Let $R$ be the spanning tree of $H$. Since $V(R) \leq 5$, every spanning tree is either a Hamilton path or a Y-tree. Then apply the above two Theorems we may find a CDC containing the circuit $C$.

\section{4 $H=G-V(C)$ is connected}

The following Lemma will be used in the proof of Theorem 2.1.9. 
Lemma 2.4.1 (Fleischner and Häggkvist [24]) For a pair $(G, C)$ with $|V(G)-V(C)| \leq$ 2 , and in the case of $|V(G)-V(C)|=2$, the distance between two vertices of $V(G)-V(C)$ is 3. (See Figure 2.7). Then $G$ has a $C D C$ containing $C$.

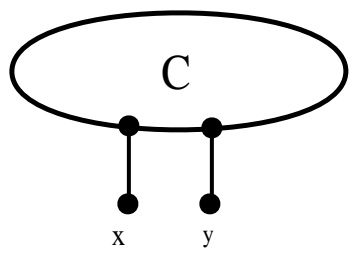

Figure 2.7: Missing two vertices

Now, we are ready to prove Theorem 2.1.9.

Proof of Theorem 2.1.9. Induction on $|V(H)|=|V(G)-V(C)|$. By Theorem 2.5.1, $g_{2}(G) \geq 9, H$ is a tree. By Corollary 2.3.6, it is sufficient to consider trees of order 6 , which are all illustrated in Figure 2.8.

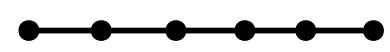

(a) Hamilton Path

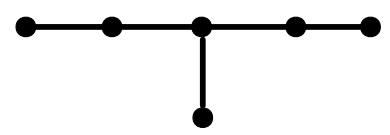

(c)

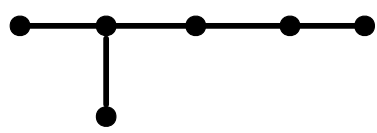

(b) Y-tree

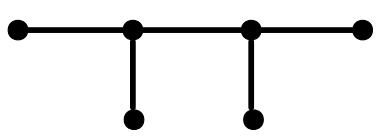

(d)

Figure 2.8: All possible spanning trees with 6 vertices

The case that $H$ is a Hamilton path (Figure $2.8(\mathrm{a})$ ) or spanning $Y$-tree (Figure 2.8 (b)) can be proved by Corollary 2.3.3 and Corollary 2.3.5.

Let $C=v_{1} v_{2} \cdots v_{r} v_{1}$ be the circuit. Since $H$ is acyclic, each leaf of $H$ must be adjacent to some vertex of $C$. Let $x_{1}$ be a leaf of $H$ such that $x_{1}$ is adjacent to a degree 2 vertex in $H$ for the case of (c) in Figure 2.8, and a leaf of $H$ for the case (d) in Figure 2.8. It 
is evident that $x_{1} v_{1} \in E(G)$ for some vertex $v_{1}$ of $C$. By the Lemma 2.2.2, either $G$ has a circuit $C^{\prime}$ with $V(C)=V\left(C^{\prime}\right)$ and $E(C) \neq E\left(C^{\prime}\right)$ or $G$ has a path $P=v_{1} v_{2} \cdots v_{j} x_{h}$ with $V(P)=V(C) \cup x_{h}$ for some vertex $x_{h} \in V(H)$, which extends $C$ to a longer circuit $C^{\prime}=v_{1} v_{2} \cdots v_{j} x_{h} \cdots x_{1} v_{1}$. In either case, the reduced pair $\left(G^{\prime}, C^{\prime}\right)$ has one of the following properties, where $G^{\prime}=\overline{G-\left(E(C)-E\left(C^{\prime}\right)\right)}$.

(1) $H^{\prime}=G^{\prime}-C^{\prime}$ remains connected and is of order at most 5 ,

(2) or $\left|V\left(H^{\prime}\right)\right|=\left|V\left(G^{\prime}\right)-V\left(C^{\prime}\right)\right|=2$ and the distance between those two vertices is 3.

By applying induction hypothesis or Lemma 2.4.1, let $\mathcal{F}^{\prime}$ be a circuit double cover of the suppressed graph $G^{\prime}$ with $C^{\prime} \in \mathcal{F}^{\prime}$. Hence, $\mathcal{F}=\mathcal{F}^{\prime}-C^{\prime}+\left\{C^{\prime} \Delta C, C\right\}$ is a circuit double cover containing the circuit $C$.

\subsection{Girth requirement for counterexample to SCDC}

The girth of a smallest counterexample to the circuit double cover was first studied by Goddyn [32], in which, a lower bound 7 of girth was found. Later, this bound was improved as follows: at least 8 by McGuinness [50], and at least 9 by Goddyn [29].

Although the CDC and SCDC are different Problems and the description of $g_{2}$ is even more complicated, proofs in some earlier articles still can be adapted for the proof of the following Lemma for SCDC problem.

Lemma 2.5.1 Let $G$ be a cubic graph, $C$ be a given circuit of $G$ and $e_{0} \in E(G-V(C))$. Assume that $G-e_{0}$ has a $C D C$ containing the given $C$ but $G$ does not. Then the edge $e_{0}$ is not contained in any circuit of $G-V(C)$ of length $\leq 8$. That is,

$$
g_{2}(G) \geq 9 .
$$

A note about the adaption of old proofs. In the studies of smallest counterexample to the CDC Conjecture (such as, [32], [50] and [29]), let $e_{0}$ be an edge of $G$ contained in 
a shortest circuit $D$. Let $\mathcal{F}$ be a $\mathrm{CDC}$ of $G-e_{0}$. And let

$$
\mathcal{F}^{\prime}=\{J \in \mathcal{F}: E(J) \cap E(D) \neq \emptyset\} .
$$

Let $G^{\prime}$ be the subgraph of $G$ induced by edges

$$
\left\{e_{0}\right\} \cup\left\{e \in J: J \in \mathcal{F}^{\prime}\right\} .
$$

In the proofs in [32], [50] and [29], by modifying circuits of $\mathcal{F}^{\prime}$ (inside of $G^{\prime}$ ), a new family $\mathcal{F}^{\prime \prime}$ of circuits of $G^{\prime}$ is obtained such that $\mathcal{F}^{\prime \prime}$ covers each edge $e$ of $G^{\prime}-\left\{e_{0}\right\}$ precisely the same as $\mathcal{F}^{\prime}$ does, and, covers the missing edge $e_{0}$ precisely twice. Hence, $\mathcal{F}-\mathcal{F}^{\prime}+\mathcal{F}^{\prime \prime}$ is a CDC of $G$.

Note that, in the proof of Lemma 2.5.1, since the shortest circuit $D$ is completely contained in $G-V(C), C \notin \mathcal{F}^{\prime}$. That is, the given circuit $C$ is not involved in the above modification, and, therefore, the given circuit $C$ remains in $\mathcal{F}-\mathcal{F}^{\prime}+\mathcal{F}^{\prime \prime}$. This explains how we are able to adapt those old proofs for Lemma 2.5.1.

Remarks. We also notice that a girth bound 10 for smallest CDC counterexample was also announced in [29], and, a computer aided result by Huck [39] further shows a best girth bound of 12 . Since we are not able to convince ourselves whether the given circuit $C$ is ever involved in the circuit covering modification, we, therefore, are only able to announce the bound $g_{2} \geq 9$ in the lemma.

\subsection{Open Problems}

Theorem 2.6.1 (Fleischner [26], also see [24]) Let $G$ be a bridgeless cubic graph of order $n$ and $C$ be a circuit of $G$ of length at least $n-1$. Then $G$ has a CDC containing the circuit $C$.

However, the following problem remains open.

Conjecture 2.6.2 (Fleischner [27]) Let $G$ be a bridgeless cubic graph of order $n$ and containing a circuit of length at least $n-1$. Then SCDC Conjecture is true for $G$. (That is, $G$ has a $C D C$ containing a circuit $C$ where $C$ is an arbitrary circuit of $G$.) 
Note that if $C$ is contained in a circuit of length $n-1$, then, by Theorem 2.6.1, $(G, C)$ has a SCDC. However, it remains open if $C$ is not contained a any circuit of length $n-1$.

Definition 2.6.3 Let $G$ be a cubic graph and $F$ be a spanning even subgraph of $G$. The oddness of $F$ is the number of odd-components of $F$. The oddness of $G$ is the minimum oddness of all spanning even subgraphs of $G$.

It is trivial that $G$ is 3-edge-colorable if and only if it is of zero oddness. Seymour proved [54] that SCDC conjecture holds for zero-oddness graphs.

Note that a graph with a Hamilton path is of oddness at most two, a graph described in Theorem 2.3.2 (containing a spanning subgraph consisting of a circuit and path) is of oddness at most four. Although the CDC conjecture have been verified for oddness two or four graphs ([40], [41], [36]), the SCDC Conjecture remains open for such small oddness graphs.

Conjecture 2.6.4 Let $G$ be a bridgeless graph of oddness at most 2. Then the SCDC conjecture is true for $(G, C)$, where $C$ is a circuit of $G$.

Conjecture 2.6.2 is obviously an extreme case of Conjecture 2.6.4.

For a specified circuit, the following is a weak version of Conjecture 2.6.4.

Conjecture 2.6.5 Let $G$ be a bridgeless graph containing a spanning even subgraph of oddness at most 2. Then the $S C D C$ conjecture is true for $(G, C)$, where $C$ is a component of $F$. 


\section{Chapter 3}

\section{Berge-Fulkerson Conjecture}

\subsection{Introduction}

In this chapter, we shall take a different view on cubic graph through perfect matching, the complement of a 2 -factor of the cubic graph.

Definition 3.1.1 A family $\mathcal{F}$ of even subgraphs of a graph $G$ is called an even subgraph $k$-cover if each edge of $G$ is contained in $k$ even subgraphs of $\mathcal{F}$.

Naturally, for an odd integer $k$, a graph $G$ has an even subgraph $k$-cover if and only if $G$ itself is an even graph. The observation comes from the symmetric difference of a family of even graphs is still even.

For the special case $k=2$, the problem of even subgraph $k$-cover is the CDC conjecture. For an even integer greater than two, we have the following results on this topic.

Theorem 3.1.2 (Bermond, Jackson and Jaeger [5]) Every bridgeless graph has a 7-even subgraph 4-cover.

Theorem 3.1.3 (Fan [16]) Every bridgeless graph has a 10-even subgraph 6-cover. 
Armed with above two theorems, it is straightforward to get the next theorem.

Theorem 3.1.4 (Fan [16]) For each even integer $k$ greater than two, every bridgeless graph has an even subgraph $k$-cover.

The following two equivalent conjectures were pointed out by Jaeger in [44].

Conjecture 3.1.5 Every bridgeless graph has a 6-even subgraph 4-cover.

Conjecture 3.1.6 Every bridgeless cubic graph has a 6-even subgraph 4-cover.

Each vertex in a cubic graph is incident to three edges, which are covered four times by the Conjecture 3.1.6, and thus belongs to each member of the covering. That means every even subgraph is spanning and therefore is a 2-factor. Note that the complement of a 2 -factor in a cubic graph is a perfect matching. We have the following equivalent statement, also known as Berge-Fulkerson Conjecture.

Conjecture 3.1.7 (Berge and Fulkerson [28]) Let $G$ be a bridgeless cubic graph. Then there exists 6 perfect matchings cover each edge of $G$ twice.

Following the definition introduced in [46] we define $m_{t}(G)$ as the maximum ratio of edges in $G$ that can be covered by $t$ perfect matchings, and by $m_{t}$ the minimum of all $m_{t}(G)$ running over all bridgeless cubic graphs. More precisely:

$$
\begin{gathered}
m_{t}(G)=\max \left\{\frac{\left|\cup_{i=1}^{t} M_{i}\right|}{|E(G)|} \mid \text { for all sets }\left\{M_{1}, \cdots, M_{t}\right\} \text { of } t \text { perfect matchings of } G\right\} ; \\
m_{t}=\inf \left\{m_{t}(G) \mid \text { for all bridgeless cubic graphs } G .\right\}
\end{gathered}
$$

By the above definition, $m_{1}=\frac{1}{3}$. Kaiser et al. [46] have proved that $m_{2}=\frac{3}{5}$, $\frac{27}{35} \leq m_{3} \leq \frac{4}{5}$. Kaiser's results in [46] implies a lower bound for $m_{5}$ is $\frac{215}{231}$, which is also proved by Mazzuccolo in [49]. Mazzuoccolo has proved that the Berge-Fulkerson Conjecture is equivalent to conjecture that $m_{5}=1$ (Berge Conjecture) [48].

Let $P_{10}$ denote the Petersen graph. They proposed the following conjecture: 
Conjecture 3.1.8 ( Kaiser, Král and Norine [46]) For $i=3,4,5, m_{i}=m_{i}\left(P_{10}\right)$.

It is proved by Patel in [51] that the Berge-Fulkerson Conjecture implies Conjecture 3.1.8. The following concepts and conjectures generalize the definitions of $m_{t}(G), m_{t}$ and Conjecture 3.1.8 for weighted cubic graphs.

Definition 3.1.9 Let $G$ be a bridgeless cubic graph associated with a weight $\omega: E(G) \mapsto$ $R^{+}$, where $R^{+}$is the set of positive real numbers. let $t$ be a positive integer. Define:

$$
\begin{gathered}
m_{t}^{*}(G ; \omega)=\max \left\{\frac{\sum_{e \in \cup_{i=1}^{t} M_{i}} \omega(e)}{\sum_{e \in E(G)} \omega(e)} \mid \text { for all sets }\left\{M_{1}, \cdots, M_{t}\right\} \text { of } t \text { perfect matchings of } G\right\} ; \\
m_{t}^{*}(G)=\inf \left\{m_{t}^{*}(G ; \omega) \mid \text { for all weights } \omega: E(G) \mapsto R^{+}\right\}
\end{gathered}
$$

and

$$
m_{t}^{*}=\inf \left\{m_{t}^{*}(G) \mid \text { for all bridgeless cubic graphs } G\right\}
$$

By their definitions, $m_{t} \geq m_{t}^{*}$. By the definition of $m_{i}^{*}, m_{1}^{*}=\frac{1}{3}$. Following the same argument in [46], we have determined that $m_{2}^{*}=m_{2}^{*}\left(P_{10}\right)=\frac{3}{5}, m_{3}^{*} \geq \frac{27}{35}, m_{4}^{*} \geq \frac{55}{63}$ and $m_{5}^{*} \geq \frac{215}{231}$ in Proposition 3.4.3. A natural problem is raised as follows: whether or not the Petersen graph $P_{10}$ attain the worst case for each $m_{t}^{*}$ ?

Conjecture 3.1.10 For $i=3,4,5, m_{i}^{*}=m_{i}^{*}\left(P_{10}\right)$.

In Proposition 3.2.4, we will show that $m_{t}^{*}\left(P_{10}\right)$ and $m_{t}\left(P_{10}\right)$ have the same value for $1 \leq t \leq 5$. Hence, the following statement is equivalent to Conjecture 3.1.10.

\section{Conjecture 3.1.10'}

$$
\text { (a) } m_{3}^{*}=\frac{4}{5} ; \quad \text { (b) } m_{4}^{*}=\frac{14}{15} ; \quad \text { (c) } m_{5}^{*}=1
$$

Obviously, Conjecture 3.1.10 implies Conjecture 3.1.8. The following is another major open problem in graph theory. 
Conjecture 3.1.11 (Fan and Raspaud [17]) Every bridgeless cubic graph contains three perfect matchings $M_{1}, M_{2}, M_{3}$ such that

$$
\bigcap_{i=1}^{3} M_{i}=\emptyset .
$$

For the case of unweighted cubic graphs, it has been proved [51] [48] that the BergeFulkerson Conjecture is equivalent to the Berge Conjecture $\left(m_{5}=1\right)$ and the Fan-Raspaud Conjecture is implied by the Berge-Fulkerson Conjecture.

In Section 3.2, some of these results for unweighted graphs are further extended. For weighted bridgeless cubic graphs, we are able to show the logical relations among those conjectures. The Berge-Fulkerson Conjecture $\left(m_{5}=1\right)$ and Conjecture 3.1.10'-(c) $\left(m_{5}^{*}=1\right)$ are equivalent to each other; And they all imply Conjecture 3.1.10'-(a) and (b) $\left(m_{3}^{*}=\frac{4}{5}\right.$ and $\left.m_{4}^{*}=\frac{14}{15}\right)$; Furthermore, each of Conjecture 3.1.10'-(a) and (b) implies the Fan-Raspaud Conjecture.

Finally, in Section 3.3, we introduce the 3-PM coverage index and prove that the determination of this index is NP-complete.

\subsection{Perfect matching covering for weighted graphs}

The following two lemmas are well-known and will be used in the proofs of this paper.

Lemma 3.2.1 Let $G$ be a bridgeless cubic graph and $T$ be an edge-cut. For any perfect matching $M$ of $G$, we have

$$
|M \cap T| \equiv|T| \quad(\bmod 2)
$$

Lemma 3.2.2 In the Petersen graph $P_{10}$, we have the following properties.

(1) $P_{10}$ has precisely 6 different perfect matchings;

(2) The intersection of each pair of distinct perfect matchings has exactly 1 edge.

(3) The intersection of any three distinct perfect matchings is empty; 
(4) Let $k$ be an integer between 2 and 5, then the union of any $k$ distinct perfect matchings has 15- $\frac{(6-k)(5-k)}{2}$ edges.

Parts (1), (2) and (3) of Lemma 3.2.2 can be seen in [54] (also see [63, 66]). Part (4) is a corollary of $(1),(2)$ and (3).

Similar to the proof of Theorem 2.1 of [51], the relation of the Berge-Fulkerson Conjecture and Conjecture 3.1.10 can be obtained as follows.

Theorem 3.2.3 The Berge-Fulkerson Conjecture implies Conjecture 3.1.10.

Since the Petersen graph $P_{10}$ has precisely six perfect matchings (by Lemma 3.2.2), the same proof of Theorem 3.2.3 can be adapted to show the following proposition.

Proposition 3.2.4

$$
m_{1}^{*}\left(P_{10}\right)=\frac{1}{3} ; m_{2}^{*}\left(P_{10}\right)=\frac{3}{5} ; m_{3}^{*}\left(P_{10}\right)=\frac{4}{5} ; m_{4}^{*}\left(P_{10}\right)=\frac{14}{15} ; m_{5}^{*}\left(P_{10}\right)=1 .
$$

\subsubsection{Fan-Raspaud Conjecture and matching coverage conjec- tures}

\section{$\oplus_{2}$-sum with Petersen graph}

The $\oplus_{2}$-sum with Petersen graph is frequently used in the following proofs.

Let $G_{1}, G_{2}$ be two bridgeless cubic graphs and $e_{1}=u v \in E\left(G_{1}\right), e_{2}=x y \in E\left(G_{2}\right)$. Construct a new graph, called the $\oplus_{2}$-sum of $G_{1}$ and $G_{2}$ associated with $e_{1}$ and $e_{2}$ (see Figure 1):

$$
G_{1} \oplus_{2} G_{2}=\left[G_{1}-\left\{e_{1}\right\}\right] \cup\left[G_{2}-\left\{e_{2}\right\}\right] \cup\{u x, v y\} .
$$

In the remainder of this paper we call those two new edges $u x, v y$, the substitution edges of $u v$ or $x y$ in the resulting graph $G_{1} \oplus_{2} G_{2}$.

Let $G$ be a cubic bridgeless graph with $m$ edges, denote by $\left\{e_{1}, e_{2}, \ldots, e_{m}\right\}$ the edge set of $G$. Let us construct a new cubic graph $G^{\prime}$ obtained by applying a $\oplus_{2}$-sum operation 


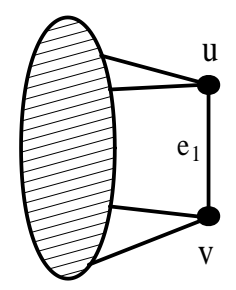

$\mathrm{G}_{1}$

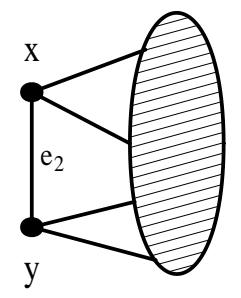

$\mathrm{G}_{2}$

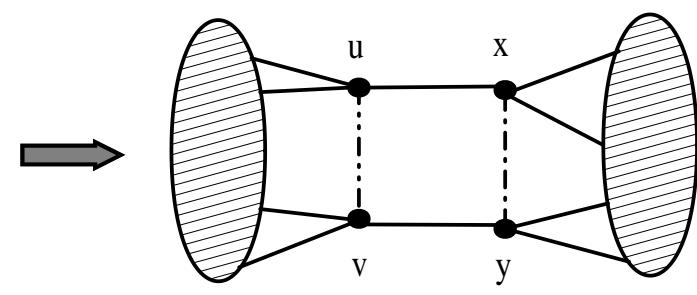

$\mathrm{G}_{1} \oplus_{2} \mathrm{G}_{2}$

Figure 3.1: $\oplus_{2}$-sum of $G_{1}, G_{2}$ associated with $e_{1}, e_{2}$

on each edge of $G$ with a copy of the Petersen graph $P_{10}$ (see Figure 2). Denote by $P_{10}^{e}$ the corresponding copy of the Petersen graph in a $\oplus_{2}$-sum operation by substituting an edge $e \in E(G)$. That is,

$$
G^{\prime}=\left(\left(\left(G \oplus_{2} P_{10}^{e_{1}}\right) \oplus_{2} P_{10}^{e_{2}}\right) \cdots \oplus_{2} P_{10}^{e_{m}}\right)
$$
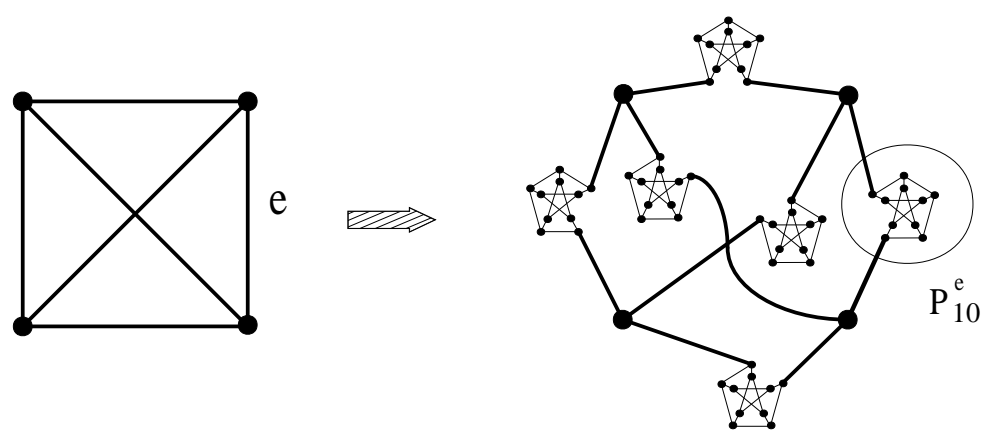

Figure 3.2: $G=K_{4}$ and the new graph $G^{\prime}$ (bold edges in $G^{\prime}$ are substitution edges)

It is evident that (1), for each $e \in E(G)$, the copy $P_{10}^{e}$ can be obtained from $G^{\prime}$ by identifying all vertices of $V\left(G^{\prime}\right)-V\left(P_{10}^{e}\right)$ and suppressing the resulting degree-2-vertices; (2) and the original graph $G$ can be obtained from $G^{\prime}$ by contracting all non-substitution-edges and suppressing all resulting degree-2-vertices. Hence, we have the following observations.

Lemma 3.2.5 Let $G$ be a cubic bridgeless graph and $G^{\prime}$ be the graph obtained by applying $\oplus_{2}$-sum operation as described above, then 
(1) For a set of perfect matchings $\left\{M_{1}^{\prime}, \ldots, M_{t}^{\prime}\right\}$ of $G^{\prime}$ and, for each $e \in E(G)$, each $M_{i}^{\prime}$ must contain both substitution edges of e or contain none of them.

(2) For each $e \in E(G)$, and each set of $t$ perfect matchings $\left\{M_{1}^{\prime}, \ldots, M_{t}^{\prime}\right\}$ of $G^{\prime}$, the corresponding set of perfect matchings $\left\{M_{1}^{e}, \ldots, M_{t}^{e}\right\}$ of $P_{10}^{e}$ can be obtained by identifying all vertices of $V\left(G^{\prime}\right)-V\left(P_{10}^{e}\right)$ and suppressing the resulting degree-2-vertices.

(3) For a set of t perfect matchings $\left\{M_{1}^{\prime}, \ldots, M_{t}^{\prime}\right\}$ of $G^{\prime}$, its corresponding set of perfect matchings $\left\{M_{1}, \ldots, M_{t}\right\}$ of $G$ can be obtained by contracting all non-substitution-edges of $G^{\prime}$ and suppressing all resulting degree-2-vertices.

Proof. Part (1) is an immediate corollary of Lemma 3.2.1. Parts (2) and (3) are straightforward conclusions of $(1)$.

\section{Fan-Raspaud Conjecture and Perfect Matching Covering}

Theorem 3.2.6 If Conjecture 3.1.10'-(a) is true for all weighted cubic graphs, then FanRaspaud Conjecture is also true.

Proof. Let $G$ be a bridgeless cubic graph. We are going to prove that there exist three perfect matchings $M_{1}, M_{2}, M_{3}$ in $G$ with the property that $\cap_{i=1}^{3} M_{i}=\emptyset$.

Define a weight function $\omega^{\prime}$ on $G^{\prime}$ (where $G^{\prime}$ is defined in Section 3.2.1) as follows:

$$
\omega^{\prime}(f)= \begin{cases}\frac{1}{2} & \text { if } f \text { is a substitution edge of an edge } e \in E(G) \\ 1 & \text { if } f \text { is not a substitution edge. }\end{cases}
$$

The total weight of $\omega^{\prime}$ on the new graph $G^{\prime}$ is $\omega^{\prime}\left(G^{\prime}\right)=15|E(G)|$. By the assumption that Conjecture 3.1.10'-(a) is true, for the newly defined weight function, there are three perfect matchings $M_{1}^{\prime}, M_{2}^{\prime}, M_{3}^{\prime}$ in $G^{\prime}$ such that $\omega^{\prime}\left(\cup_{i=1}^{3} M_{i}^{\prime}\right) \geq \frac{4}{5} \omega^{\prime}\left(G^{\prime}\right)=12|E(G)|$.

Each edge of $G$, after a $\oplus_{2}$-sum operation, is replaced by a 2-edge cut consisting of its two substitution edges. By Lemma 3.2.1, every perfect matching intersects every such 2-edge cut an even number of time. Let $E_{0}$ be the set of edges in $G$ whose two substitution edges in $G^{\prime}$ are covered by all three perfect matchings $\left\{M_{1}^{\prime}, M_{2}^{\prime}, M_{3}^{\prime}\right\}$. 
Let $\left\{M_{1}, M_{2}, M_{3}\right\}$ be the set of perfect matchings of $G$ corresponding to $\left\{M_{1}^{\prime}, M_{2}^{\prime}, M_{3}^{\prime}\right\}$ (see Lemma 3.2.5-(3)). Here, $E_{0}=\bigcap_{i=1}^{3} M_{i}$, and we are to show that $E_{0}=\emptyset$.

For each $e \in E_{0}=\bigcap_{i=1}^{3} M_{i}$, let $\left\{M_{1}^{e}, M_{2}^{e}, M_{3}^{e}\right\}$ be the set of perfect matchings of $P_{10}^{e}$ corresponding to $\left\{M_{1}^{\prime}, M_{2}^{\prime}, M_{3}^{\prime}\right\}$ (see Lemma 3.2.5-(2)). Therefore, those three perfect matchings in $P_{10}^{e}$ intersect in at least one edge and $\left|\left\{M_{1}^{e}, M_{2}^{e}, M_{3}^{e}\right\}\right| \leq 2$ since there are no three distinct perfect matchings in Petersen graph with nonempty intersection by Lemma 3.2.2-(2).

By Lemma 3.2.2-(4), two distinct perfect matchings cover exactly 9 edges of the Petersen graph and three distinct perfect matchings cover exactly 12 edges, we have that

$$
\omega^{\prime}\left(\cup_{i=1}^{3} M_{i}^{\prime}\right) \leq 9\left|E_{0}\right|+12\left(|E(G)|-\left|E_{0}\right|\right)=12|E(G)|-3\left|E_{0}\right| .
$$

Then, by the fact that $\omega^{\prime}\left(\cup_{i=1}^{3} M_{i}^{\prime}\right) \geq 12|E(G)|$, we have $\left|E_{0}\right|=0$, as we desired.

Theorem 3.2.7 If Conjecture 3.1.10'-(b) is true for all weighted cubic graphs, then FanRaspaud Conjecture is also true.

Proof. The proof is similar to the argument used in above Theorem. The only difference is the definition of edge set $E_{0}$, which consisting of all edges in $G$ whose two substitution edges are covered by three or four perfect matchings in $G^{\prime}$. We shall get a similar inequality

$$
14|E(G)| \leq \omega^{\prime}\left(\cup_{i=1}^{4} M_{i}^{\prime}\right) \leq 12\left|E_{0}\right|+14\left(|E(G)|-\left|E_{0}\right|\right)
$$

which implies that $\left|E_{0}\right|=0$, as we desired.

\section{3 $3 P M$-Coverage Index - a rank for snarks}

Definition 3.3.1 Let $G$ be a bridgeless cubic graph, we call $m_{3}(G)$ the $3 P M$-coverage index of $G$, denoted by $\tau(G)$; Similarly we call $m_{3}^{*}(G)$ the weighted $3 P M$-coverage index of $G$, denoted by $\tau^{*}(G)$.

According to Conjectures 3.1.8 and 3.1.10'-(a), the lower bound for both $\tau$ and $\tau^{*}$ is expected to be at least $\frac{4}{5}$. A graph has the $3 P M$-coverage index $\tau=1$ if and only if it is 
3-edge-colorable. Hence, the graph invariant $\tau$ (as well as its weighted version $\tau^{*}$ ) can be considered as a ranking for snarks.

The following figure (Figure 3.3) illustrates two examples with 3PM-Coverage indices between $\frac{4}{5}$ and 1 . For the Tietze's snark $T, \tau(T)=\frac{5}{6}$ and, for the Blanuša snark $B$, $\tau(B)=\frac{8}{9}$.

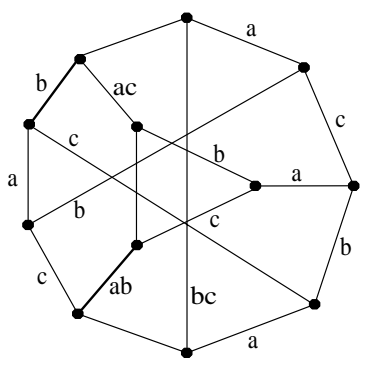

(a) Tietze's snark

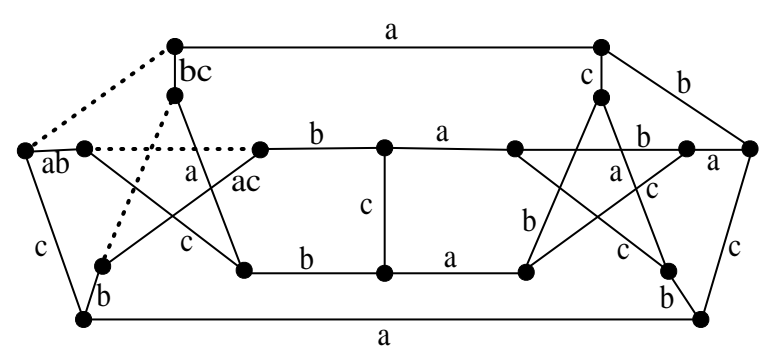

(b) Blanuša snark

Figure 3.3: Two snarks with 3PM-Coverage indices $\frac{5}{6}$ and $\frac{8}{9}$ respectively

In Figure 3.3, each snark has a set of 3 perfect matchings $\left\{M_{a}, M_{b}, M_{c}\right\}$ where edges labeled with $x(x \in\{a, b, c\})$ belong to the matching $M_{x}$, and dashed edges are not covered by $M_{a} \cup M_{b} \cup M_{c}$.

Just like the determination of 3-edge-colorability [38], the determination of the invariant $\tau^{*}$ for $\tau^{*}>\frac{4}{5}$ is also an $N P$-complete problem (see Theorem 3.3.3).

Instance. A bridgeless cubic graph $G$ associated with a positive weight $\omega: E(G) \mapsto R^{+}$, and a real number $\eta: \frac{4}{5}<\eta<1$.

Problem 3.3.2 Does the graph $G$ have a set of three perfect matchings $\left\{M_{1}, M_{2}, M_{3}\right\}$ with the property that $\omega\left(\cup_{i=1}^{3} M_{i}\right) \geq \eta \omega(G)$ ?

Theorem 3.3.3 Question 3.3.2 is an NP-Complete problem.

Proof. It is known that the 3-edge-coloring problem is an NP-complete problem [38]. In this proof, we will use contradiction to prove this theorem. Let us suppose that the problem is not an NP-Complete problem, then there exists a polynomial time algorithm 
that determines for any cubic bridgeless graph $H$, whether there exists a set of 3 perfect matchings $\left\{M_{1}, M_{2}, M_{3}\right\}$ of $H$ such that $\omega\left(\cup_{i=1}^{3} M_{i}\right) \geq \eta \omega(H)$ where $1>\eta>\frac{4}{5}$. In the following we will show such a polynomial time algorithm can be used to construct a polynomial time algorithm to determine if a cubic bridgeless graph $G$ is 3-edge-colorable.

Since $\eta>\frac{4}{5}$, there exists an $\epsilon>0$ such that $\eta=\frac{12+1.2 \epsilon}{15}$.

Let $G$ be a bridgeless cubic graph with $m$ edges, and $G^{\prime}$ be as defined in Section 3.2.1. Define the weight function $\omega^{\prime}$ on $G^{\prime}$ as follows.

$$
\omega^{\prime}(f)= \begin{cases}\frac{1}{2} & \text { if } f \text { is a substitution edge of some } e \in E(G) \\ 1+\epsilon & \text { if } f \text { is incident with a substitution edges of } e \\ 1-0.4 \epsilon & \text { else }\end{cases}
$$

Therefore, $\omega^{\prime}\left(G^{\prime}\right)=15 m$.

Since $G^{\prime}$ is also a bridgeless cubic graph, the polynomial time algorithm can determine if there exist 3 perfect matchings $\left\{M_{1}^{\prime}, M_{2}^{\prime}, M_{3}^{\prime}\right\}$ covering all edges of $G^{\prime}$ such that $\omega^{\prime}\left(\cup_{i=1}^{3} M_{i}^{\prime}\right) \geq \eta\left(G^{\prime}\right)=\frac{12+1.2 \epsilon}{15} \omega^{\prime}\left(G^{\prime}\right)=(12+1.2 \epsilon) m$

Case 1. If the polynomial time algorithm determines that there exist three perfect matchings $\left\{M_{1}^{\prime}, M_{2}^{\prime}, M_{3}^{\prime}\right\}$ of $G^{\prime}$ such that $\omega^{\prime}\left(\cup_{i=1}^{3} M_{i}^{\prime}\right) \geq \eta\left(G^{\prime}\right)=\frac{12+1.2 \epsilon}{15} \omega^{\prime}\left(G^{\prime}\right)=(12+$ $1.2 \epsilon) \mathrm{m}$, then we will prove that the algorithm can be used to find a 3-edge coloring of $G$.

By Lemma 3.2.5-(3), let $\left\{M_{1}^{e}, M_{2}^{e}, M_{3}^{e}\right\}$ be the corresponding set of perfect matchings in $P_{10}^{e}$ for an edge $e \in E(G)$.

Claim For each edge $e \in E(G), \omega^{\prime}(x)=1$ where $x$ is the edge of $P$ arisen from two substitution edges and

$$
\sum_{f \in P_{10}^{e} \cap\left(\cup_{i=1}^{3} M_{i}^{\prime}\right)} \omega^{\prime}(f)=\sum_{f \in \cup_{i=1}^{3} M_{i}^{e}} \omega^{\prime}(f) \leq(12+1.2 \epsilon) .
$$

The equality holds if and only if each substitution edge of e and all edges in $P_{10}^{e}$ incident with substitution edges of e are covered precisely once by $\left\{M_{1}^{\prime}, M_{2}^{\prime}, M_{3}^{\prime}\right\}$.

Proof of the Claim. By Lemma 3.2.2, the set of perfect matchings $\left\{M_{1}^{e}, M_{2}^{e}, M_{3}^{e}\right\}$ covers at most 12 edges of $P_{10}^{e}$. So the total weight of the edges they covered is at most

$$
15-3(1-0.4 \epsilon)=12+1.2 \epsilon
$$


since a least weighted edge is of weight $(1-0.4 \epsilon)$. Notice that those least weighted edges are not incident with substitution edges of $e$. The total weight $\omega^{\prime}\left(\bigcup_{i=1}^{3} M_{i}^{e}\right)$ reaches its maximum if and only if $\left\{M_{1}^{e}, M_{2}^{e}, M_{3}^{e}\right\}$ are distinct in $P_{10}^{e}$ and misses precisely three edges of weight $(1-0.4 \epsilon)$. (Figure 4 is an example showing a set of three perfect matchings $\left\{M_{a}, M_{b}, M_{c}\right\}$ of $P_{10}^{e}$ missing three dashed edges with weight $(1-0.4 \epsilon)$.) This completes the proof of the Claim.

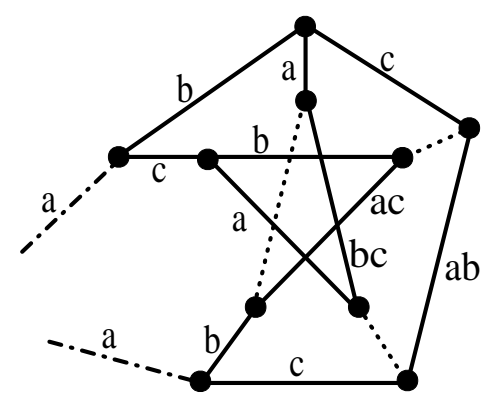

Figure 3.4: Three perfect matchings $\left\{M_{a}, M_{b}, M_{c}\right\}$ reach the maximum coverage in $P_{10}^{e}$.

Since $\omega^{\prime}\left(\cup_{i=1}^{3} M_{i}^{\prime}\right) \geq \frac{12+1.2 \epsilon}{15} \omega^{\prime}\left(G^{\prime}\right)=(12+1.2 \epsilon) m$, by the above Claim, each corresponding $\left\{M_{1}^{e}, M_{2}^{e}, M_{3}^{e}\right\}$ must reach its maximum total weight in $P_{10}^{e}$. Thus, we have that

(1) Each substitution edge of $e$ is covered once in $G^{\prime}$

(2) All edges in $P_{10}^{e}$ incident to substitution edges are also covered once.

By Lemma 3.2.5, the corresponding set of perfect matchings $\left\{M_{1}, M_{2}, M_{3}\right\}$ of $G$ is a 1 -factorization of $G$. This way we can find a 3-edge coloring of the graph $G$.

Case 2. If the polynomial time algorithm determines that any set of 3 perfect matchings of $G^{\prime}$ doesn't have the property. Then we claim that $G$ is not 3-edge colorable.

Suppose not, let $\left\{M_{1}, M_{2}, M_{3}\right\}$ be a 1-factorization of $G$. Then we can construct 3 distinct perfect matchings $\left\{M_{1}^{\prime}, M_{2}^{\prime}, M_{3}^{\prime}\right\}$ of $G^{\prime}$ just as in the above analysis. It's easy to check that the total weight of edges covered by the 3 perfect matching is of $\eta$ ratio of the total weight of $G^{\prime}$, a contradiction. 
By the above analysis, if Question 3.3.2 is solvable with a polynomial time algorithm, then the algorithm can be used to determine if a cubic bridgeless graph is 3-edge-colorable. A contradiction to the fact that determining if a cubic bridgeless graph is 3-edge-colorable is an NP-Complete problem. Thus Question 3.3.2 is an NP-complete problem.

\subsection{Remarks}

\section{NP-completeness of the invariant $\tau$}

Theorem 3.3.3 shows that the determination of the invariant $\tau^{*}$ is an NP-complete problem. However, the computational complexity of the unweighted invariant $\tau$ remains unknown.

Conjecture 3.4.1 Given a real number $1>\eta>\frac{4}{5}$, the decision problem whether $\tau(G) \geq$ $\eta$ for an unweighted cubic bridgeless graph $G$ is also an NP-complete problem.

\section{Shortest cycle covers and the invariant $\tau$}

An even subgraph of a graph $G$ is a subgraph such that all the degrees of its vertices are even. A 3-even subgraph cover of $G$ is a set $\left\{F_{1}, F_{2}, F_{3}\right\}$ of three even subgraphs such that every edge of $G$ is contained in at least one of $\left\{F_{1}, F_{2}, F_{3}\right\}$. In [17], it is proved that Conjecture 3.1.11 implies that every bridgeless cubic graph $G$ has a 3-even subgraph cover with total length at most $\frac{22|E(G)|}{15}$. The following is a problem proposed from that result and some observations about the invariant $\tau$.

Problem 3.4.2 Is there a non-increasing function $f:\left[\frac{4}{5}, 1\right] \mapsto\left[\frac{4}{3}, \frac{22}{15}\right]$ such that every graph with $\tau(G)=t$ has a 3 -even subgraph cover of total length at most $f(t)|E(G)|$ ?

\section{Some results about the invariants $m_{i}^{*}$.}

In [46], et al. have proved that $m_{2}=m_{2}\left(P_{10}\right)=\frac{3}{5}, \frac{27}{35} \leq m_{3} \leq \frac{4}{5}$. Mazzuoccolo [49] further determined that $\frac{215}{231} \leq m_{5}$. The proofs of these results can be modified for weighted cubic graphs as follows. 
Proposition 3.4.3

$$
\begin{aligned}
& m_{1}^{*}=m_{1}^{*}\left(P_{10}\right)=\frac{1}{3} ; \quad m_{2}^{*}=m_{2}^{*}\left(P_{10}\right)=\frac{3}{5} \\
& \frac{27}{35} \leq m_{3}^{*} \leq \frac{4}{5} ; \quad \frac{55}{63} \leq m_{4}^{*} ; \quad \frac{215}{231} \leq m_{5}^{*}
\end{aligned}
$$

\subsection{Appendix}

In this appendix, we prove Proposition 3.4.3.

Proposition 3.4.3

$$
\begin{aligned}
& m_{1}^{*}=m_{1}^{*}\left(P_{10}\right)=\frac{1}{3} ; \quad m_{2}^{*}=m_{2}^{*}\left(P_{10}\right)=\frac{3}{5} \\
& \frac{27}{35} \leq m_{3}^{*} \leq \frac{4}{5} ; \quad \frac{55}{63} \leq m_{4}^{*} ; \quad \frac{215}{231} \leq m_{5}^{*}
\end{aligned}
$$

\subsubsection{The perfect matching polytope}

Let $G$ be a graph. An edge subset $C$ of $G$ is called an edge cut if $G-C$ has more components than $G$ does, and $C$ is inclusion-wise minimal with this property. A $k$-cut is an edge cut of cardinality $k$. Let $S$ be a vertex subset of $G$, we denote by $\partial S$ the set of edges incident to exactly one vertex in $S$. Let $\omega$ be a vector in $R^{E(G)}$. The entry of $\omega$ corresponding to an edge $e$ is denoted by $\omega(e)$, and for $A \subset E(G)$, we define the weight $\omega(A)$ of $A$ as $\sum_{e \in A} \omega(e)$. The vector $\omega$ is said to be a fractional perfect matching of $G$ if it satisfies the following properties:

(a) $0 \leq \omega(e) \leq 1$, for each $e \in E(G)$,

(b) $\omega(\partial\{v\})=1$ for each vertex $v \in V$, and

(c) $\omega(\partial X) \geq 1$ for each $X \subset V(G)$ of odd cardinality.

Given an edge subset $F \subset E(G), \chi^{F} \in R^{E(G)}$ is defined as follows: $\chi^{F}(e)=1$ if and only if $e \in F$. 
The proof of our results is based on the following fundamental theorem in graph theory.

Lemma 3.5.1 (Edmonds [14]) If $\omega$ is a fractional perfect matching in a graph $G$ and $c \in R^{E(G)}$, then $G$ has a perfect matching $M$ such that

$$
c \cdot \chi^{M} \geq c \cdot \omega
$$

where denotes the scalar product. Moreover, there exists such a perfect matching $M$ that contains exactly one edge of each cut $C$ with $\omega(C)=1$.

\section{Lemma 3.5.2}

$$
m_{1}^{*}=\frac{1}{3}, \quad m_{2}^{*}=\frac{3}{5}
$$

Proof. By our discussion in main part of the paper, it suffices to prove that for any cubic bridgeless graph $G$ with any positive weight function $\omega$ we have

$$
m_{1}(G ; \omega) \geq \frac{1}{3}, \quad m_{2}(G ; \omega) \geq \frac{3}{5}
$$

Define $c_{1}, \omega_{1} \in R^{E(G)}$ such that $c_{1}$ has value $\omega(e)$ and $\omega_{1}$ has the value $1 / 3$ on each $e \in E$. It is easy to verify that $\omega_{1}$ is a fractional perfect matching of $G$. Moreover, $\omega_{1}(C)=1$ for each 3-cut $C$ of $G$. Hence, by Lemma 3.5.1, there is a perfect matching $M_{1}$ intersecting each 3-cut in a single edge such that $\omega\left(M_{1}\right)=c_{1} \cdot \chi^{M_{1}} \geq c_{1} \cdot \omega=\frac{1}{3} \omega(G)$. So $m_{1}(G ; \omega) \geq \frac{1}{3}$. Thus $m_{1}^{*}=\frac{1}{3}$.

We now use $M_{1}$ to define the following vector $\omega_{2} \in R^{E(G)}: \omega_{2}(e)= \begin{cases}\frac{1}{5} & \text { if } e \in M_{1} \\ \frac{2}{5} & \text { otherwise }\end{cases}$

Again, it can be verified that $\omega_{2}$ is a fractional perfect matching of $G$ (it is important that $M_{1}$ contains exactly one edge of each 3-cut of $\left.G\right)$. For each $e \in E$, set $c_{2}(e)=(1-$ $\left.\chi^{M_{1}}(e)\right) \omega(e)$. By Lemma 3.5.1, there exists a perfect matching $M_{2}$ such that $c_{2} \cdot \chi^{M_{2}} \geq c_{2}$. $\omega_{2}=\frac{2}{5}\left(\omega(G)-\omega\left(M_{1}\right)\right)$. Since $c_{2} \cdot \chi^{M_{2}}$ is just $\omega\left(M_{2}\right)-\omega\left(M_{1}\right)$, it follows that $\omega\left(M_{1} \bigcup M_{2}\right)=$ $\omega\left(M_{1}\right)+\left(\omega\left(M_{2}\right)-\omega\left(M_{1}\right)\right) \geq \omega\left(M_{1}\right)+\frac{2}{5}\left(\omega(G)-\omega\left(M_{1}\right)\right)=\frac{2}{5} \omega(G)+\frac{3}{5} \omega\left(M_{1}\right) \geq \frac{3}{5} \omega(G)$. We conclude that $m_{2}^{*}(G ; \omega) \geq \frac{3}{5}$. So $m_{2}^{*}=\frac{3}{5}$. 
Lemma 3.5.3 For any bridgeless cubic graph $G$ with a positive weight function $\omega$, let $t$ be a positive integer between 1 and 5 and $a_{t}$ be the sequence defined by the law $a_{t}=$ $\frac{t}{2 t+1}\left(1-a_{t-1}\right)+a_{t-1}$ and $a_{0}=0$, we have $m_{t}(G ; \omega) \geq a_{t}$.

Proof. In [49], Mazzuoccolo further studied the problem of covering a bridgeless cubic graph with perfect matchings. The following proof is similar to his proof, here we just mention some parts that is important and different from his proof.

By Lemma 3.5.2, the proposition holds for $t=1,2$, we will use induction to prove this. Suppose the proposition holds for $t$, in the following we will prove that it holds for $t+1$.

Let $M^{t}=\left\{M_{1}, \ldots, M_{t}\right\}$ be a set of $t$ perfect matchings, define a weight $\omega_{M^{t}}$ of $G$ as follows:

$$
\omega_{M^{t}}(e)=\frac{t+1-\sum_{i=1}^{t}\left|M_{i} \bigcap\{e\}\right|}{2 t+3}
$$

Observe that when $t=0,1$, we obtain $\omega_{1}, \omega_{2}$ as defined in Lemma 3.5.2 and they are both fractional perfect matching of $G$.

Let $M^{t}=\left\{M_{1}, \ldots, M_{t}\right\}$ be a set of $t$ perfect matchings such that $\omega_{M^{t}}$ is a fractional perfect matching of $G$. For each $e \in E$, set $c_{t}(e)=\left(1-\chi^{\bigcup_{i=1}^{t} M_{i}(e)}\right) \omega(e)$. By Lemma 3.5.1, there exists a perfect matching $M_{t+1}$ such that

$$
c_{t} \cdot \chi^{M_{t+1}} \geq c_{t} \cdot \omega_{M^{t}}
$$

and $M_{t+1}$ contains exactly one edge for each cut $C$ with $\omega_{M^{t}}(C)=1$. In [49], G.Mazzuoccolo proved that $\omega_{M^{t+1}}$, where $M^{t+1}=M^{t} \bigcup\left\{M_{t+1}\right\}$, is a fractional perfect matching of $G$.

By Lemma 3.5.2, the basic step $\omega_{M^{0}}=\frac{1}{3}$ is trivially a fractional perfect matching, we have that $\omega_{M^{t}}$ (with $M^{t}$ constructed as described above) is a fractional perfect matching for each value of $t$. Therefore, the following holds for each positive integer $t$ :

$$
c_{t} \cdot \chi^{M_{t+1}} \geq c_{t} \cdot \omega_{M^{t}}
$$

The left side of the previous inequality is exactly the total weights of edges of $M_{t+1}$ not covered by $M^{t}$, while the right side is $\frac{t+1}{2 t+3}$ times the total weights of edges not covered 
by $M^{t}$. Denoting by $b_{t}$ the fraction of the total weights of edges covered by $M^{t}$, we obtain

$$
\begin{gathered}
b_{t+1}-b_{t} \geq\left(1-b_{t}\right) \cdot \frac{t+1}{2 t+3} \\
m_{t+1}(G ; \omega) \geq b_{t+1} \geq\left(1-b_{t}\right) \cdot \frac{t+1}{2 t+3}+b_{t}=\frac{t+1+(t+2) b_{t}}{2 t+3} \geq \frac{t+1+(t+2) a_{t}}{2 t+3}=a_{t+1}
\end{gathered}
$$

and the assertion follows.

By Lemma 3.5.3, the following theorem follows.

Theorem 3.5.4 Let $a_{t}$ be defined as above, then $m_{t}^{*} \geq a_{t}$.

By a straightforward calculation, $a_{3}=\frac{27}{35}, a_{4}=\frac{55}{63}, a_{5}=\frac{215}{231}$ and the following corollary holds:

\section{Corollary 3.5.5}

$$
m_{3}^{*} \geq \frac{27}{35}, \quad m_{4}^{*} \geq \frac{55}{63}, \quad m_{5}^{*} \geq \frac{215}{231} .
$$




\section{Chapter 4}

\section{Saturation Number for Paths}

\subsection{Introduction and Notation}

A fixed graph $G$ is called an $H$-saturated graph if the graph $H$ is not a subgraph of $G$, but adding any missing edge to $G$ will produce a copy of $H$. The collection of all $H$-saturated graphs of order $n$ is denoted by $S A T(n, H)$, and the saturation number, denoted $\operatorname{sat}(n, H)$, is the minimum number of edges of a graph in the set $S A T(n, H)$. The graphs in $S A T(n, H)$ with the minimum number of edges will be denoted by $\underline{S A T}(n, H)$. The saturation number was introduced by Erdős, Hajnal, and Moon in [15] in which the authors proved $\operatorname{sat}\left(n, K_{p}\right)=\left(\begin{array}{c}p-2 \\ 2\end{array}\right)+(n-p+2)(p-2)$ and $\underline{S A T}\left(n, K_{p}\right)=\left\{K_{p-2}+\bar{K}_{n-p+2}\right\}$, where + is the standard graph joining operation. The maximum number of edges of a graph from $\operatorname{SAT}(n, H)$ is the well known Turán extremal number (see [59]), and is usually denoted by $e x(n, H)$. The parameters $\operatorname{sat}(n, H)$ and $e x(n, H)$ have been investigated for a range of graphs $H$. Generalization to hypergraphs also exist (see [52]).

A natural question is to find, if possible, an $H$-saturated graph with $m$ edges for any integer $m$ between the saturation number and extremal number. Barefoot et. al [4] studied the edge spectrum of $K_{3}$-saturated graphs and proved the following result.

Theorem 4.1.1 [4] Let $n \geq 5$ and $m$ be nonnegative integers. There is an $(n, m) K_{3}$ saturated graph if and only if $2 n-5 \leq m \leq\left\lfloor(n-1)^{2} / 4\right\rfloor+1$ or $m=k(n-k)$ for some 
positive integer $k$.

Theorem 4.1.1 says that a $K_{3}$-saturated graph is either a complete bipartite graph or its size falls in the given range and all values in this range are possible. Later Amin [2] extended this result from $K_{3}$-saturated graphs to any complete graph $K_{p}$ at her Ph.D. thesis, which is the starting point of this chapter.

\subsection{Known Results about Extremal Number on Paths}

In [33] Kászonyi and Tuza proved several general results concerning saturated graphs including an upper bound for $\operatorname{sat}(n, H)$ for any connected graph $H$ by constructing an $H$-saturated graph.

Theorem 4.2.1 [33] Saturation Numbers for Paths:

(a) For $n \geq 3$, sat $\left(n, P_{3}\right)=\lfloor n / 2\rfloor$.

(b) For $n \geq 4$, sat $\left(n, P_{4}\right)= \begin{cases}n / 2 & \text { if } n \text { is even, } \\ (n+3) / 2 & \text { if } n \text { is odd. }\end{cases}$

(c) For $n \geq 5$, sat $\left(n, P_{5}\right)=\left\lceil\frac{5 n-4}{6}\right\rceil$.

(d) Let $a_{k}=\left\{\begin{array}{l}3 \cdot 2^{t-1}-2 \quad \text { if } k=2 t, \\ 4 \cdot 2^{t-1}-2 \quad \text { if } k=2 t+1 .\end{array}\right.$ If $n \geq a_{k}$ and $k \geq 6$, then $\operatorname{sat}\left(n, P_{k}\right)=$ $n-\left\lfloor\frac{n}{a_{k}}\right\rfloor$.

In this paper, we will mainly consider the case of $P_{5}$ and $P_{6}$. By Theorem 4.2 .1 we have $\operatorname{sat}\left(n, P_{6}\right)=\lceil 9 n / 10\rceil$ for any $n \geq 10$.

Next let us recall a result about the Turán extremal number, which was proved by Faudree and Schelp [18] in 1975.

Theorem 4.2.2 [18] If $G$ is a graph with $|V(G)|=k t+r, 0 \leq r<k$, containing no path on $k+1$ vertices, then $|E(G)| \leq t\left(\begin{array}{c}k \\ 2\end{array}\right)+\left(\begin{array}{c}r \\ 2\end{array}\right)$ with equality if and only if $G$ is either 
$\left(t K_{k}\right) \cup K_{r}$ or $\left((t-l-1) K_{k}\right) \cup\left(K_{(k-1) / 2}+\bar{K}_{(k+1) / 2+l k+r}\right)$ for some $l, 0 \leq l<t$, where $k$ is odd, $t>0$, and $r=(k \pm 1) / 2$.

Corollary 4.2.3 [55] For all integer $n, n \geq 3$,

(a) $\operatorname{ex}\left(n, P_{4}\right)= \begin{cases}n & n \equiv 0(\bmod 3) \\ n-1 & n \equiv 1,2(\bmod 3)\end{cases}$

(b) $\operatorname{ex}\left(n, P_{5}\right)= \begin{cases}3 n / 2 & n \equiv 0(\bmod 4) \\ 3 n / 2-2 & n \equiv 2(\bmod 4) \\ 3(n-1) / 2 & n \equiv 1,3(\bmod 4)\end{cases}$

(c) $\operatorname{ex}\left(n, P_{6}\right)= \begin{cases}2 n & n \equiv 0(\bmod 5) \\ 2 n-2 & n \equiv 1,4(\bmod 5) \\ 2 n-3 & n \equiv 2,3(\bmod 5) .\end{cases}$

Considering the fact that for any $P_{3}$-saturated graph $G$, no two edges can be incident to each other and $G$ contains at most one isolated vertex, therefore $\operatorname{sat}\left(n, P_{3}\right)=$ ex $\left(n, P_{3}\right)=\lfloor n / 2\rfloor$. As for the case of $P_{4}$-saturated graphs, the following figures clearly show how we can evolve a $P_{4}$-saturated with least edges to most ones.

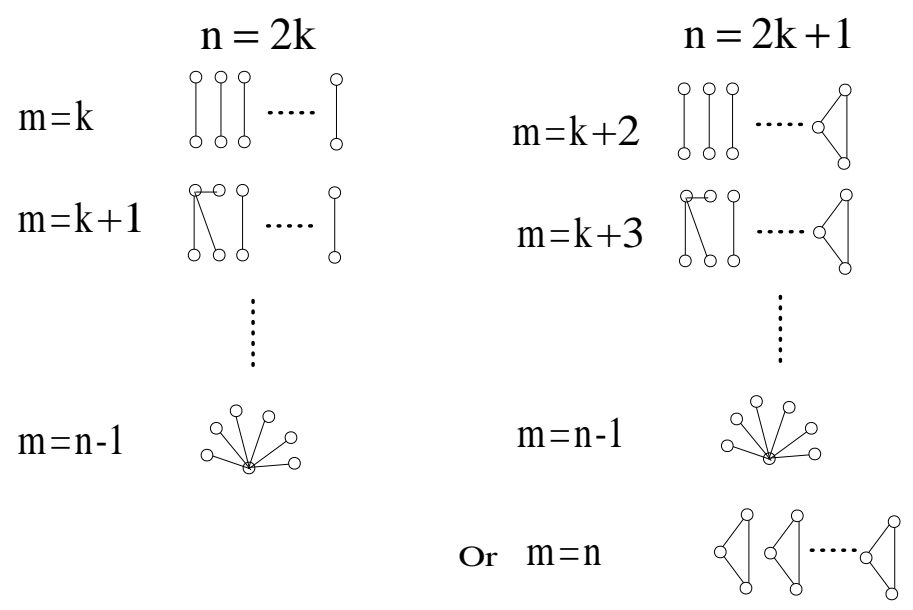

Figure 4.1: Description of $P_{4}$-saturated graphs 
From the Figure 4.1 we can evolve a $P_{4}$-saturated graph, a perfect matching or a matching union a triangle, to one with $n-1$ edges for any integer $n$. In addition, when $n=3 p$ we can take $G=p K_{3}$ and find one more $P_{4}$-saturated graph of size $n$.

\subsection{Edge Spectrum of $P_{5}$-Saturated Graphs}

If $G$ is a $P_{5}$-saturated graph with order less than 5 , then $G$ must be a complete graph. What's more, the order of the union of any two components of $G$ must be at least 5 since otherwise we can add an edge joining those two components and have no copies of $P_{5}$ in the resulting graphs. Therefore the structure of every component of a $P_{5}$-saturated graph becomes important and we have the following lemma concerning it.

Lemma 4.3.1 If $H$ is a connected $P_{5}$-saturated graph with order at least 5, then each block of $H$ is a clique of order 2 or 3.

Proof. If $H$ is 2-connected, i.e. $H$ consists of only one block, $H$ can not be a complete graph since $H$ is $P_{5}$-saturated graph. Hence we may take two vertices $u$ and $v$ with $u v \notin E(H)$. There is no circuit with length more than 4 since otherwise a subgraph $P_{5}$ will be found. Therefore, we can find a circuit of length 4 . Considering that $n \geq 5$, there is a vertex $w$ connecting to some vertex on this circuit and forming a path $P_{5}$. Thus $H$ can not be 2-connected.

Let $B$ be a block of $H$, then the order of $B$ must be less than 4 since otherwise we may find a circuit of length at least 4 within block $B$, which forms a $P_{5}$ with any one edge outside $B$. Thus the order of each block in $H$ is either 3 or 2 , as we desired.

Let $H$ be a connected $P_{5}$-saturated graph. It is trivial for the case $|V(H)| \leq 4$ that $H$ must be a complete graph. Hence we may assume that $|V(H)| \geq 5$. If $H$ contains two triangles $T_{1}$ and $T_{2}$, which are connected by a path $Q$, then a path of order at least 5 is naturally contained as a subgraph. Hence $H$ contains at most one triangle. Therefore, by Lemma 4.3.1 either $G$ is a tree or a graph obtained by adding exactly one edge to a star (See Figure 4.2 ). Then we get the following Lemmas. 

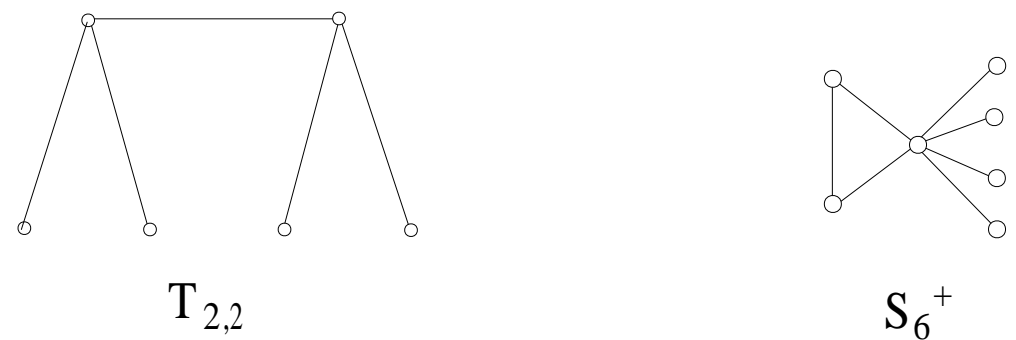

Figure 4.2: Two possible structures for a connected $P_{5}$-saturated graph

Lemma 4.3.2 If $H$ is a component of $P_{5}$-saturated graph other than $K_{4}$, then the size of $H$ is either $n^{\prime}-1$ or $n^{\prime}$, where $n^{\prime}=|V(H)|$.

Let $G$ be a $P_{5}$-saturated graph and $S_{\mu}^{+}$be a graph obtained from the star $S_{\mu}$ by adding one edge. If we denote by $\alpha_{1}$ the number of acyclic components of $G, \alpha_{2}$ the number of $S_{\mu}^{+}$'s, $\alpha_{3}$ the number of $K_{4}^{\prime} s$ in $G$, then by applying the above lemma we have the next lemma immediately.

Lemma 4.3.3 If $G \in S A T\left(n, P_{5}\right)$, then $|E(G)|=n-\alpha_{1}+2 \alpha_{3}$, where $\alpha_{1}$, $\alpha_{3}$ are defined above.

The key idea of our method is constructing a new $P_{5}$-saturated graph from an existing smaller $P_{5}$-saturated graph. Our main result on $P_{5}$ is heavily dependent on the following lemmas.

Lemma 4.3.4 Let $m \geq n \geq 5$. There is an $(n, m)$ graph $G$ in $S A T\left(n, P_{5}\right)$ if and only if there exists an $(n+4, m+6)$ graph $G^{\prime}$ in $S A T\left(n+4, P_{5}\right)$.

Proof. It is trivial that $G \in S A T\left(n, P_{5}\right)$ implies $G^{\prime}=G \cup K_{4} \in S A T\left(n+4, P_{5}\right)$. For the necessity, we assume that $G^{\prime}$ is an $(n+4, m+6)$ graph in $S A T\left(n+4, P_{5}\right)$. If there exists a component $K_{4}$ in $G^{\prime}$, then $G=G^{\prime}-K_{4}$ will be an $(n, m)$ graph in $S A T\left(n, P_{5}\right)$. Hence, we may suppose that $G^{\prime}$ contains no $K_{4}$ 's, i.e. $\alpha_{3}=0$. By Lemma 4.3.3, $m+6=\left|E\left(G^{\prime}\right)\right|=\left|V\left(G^{\prime}\right)\right|-\alpha_{1} \leq\left|V\left(G^{\prime}\right)\right|=n+4$, contradicting that $m \geq n$.

So far we have figured out $P_{5}$-saturated graph with $m \geq n$. The next lemma will tell us more information about $P_{5}$-saturated graph with less edges. 
Lemma 4.3.5 Let $n$ be an integer at least 5 and $\left\lceil\frac{5 n-4}{6}\right\rceil \leq m \leq n-1$. There exists a $P_{5}$-saturated $(n, m)$ graph.

Proof. If $n=5$, then $\left\lceil\frac{5 n-4}{6}\right\rceil=n-1=4$ and take $G=K_{2} \cup K_{3} \in S A T\left(n, P_{5}\right)$. Next we assume that $n>5$ and write $n=6 k+i$, where $0 \leq i \leq 5$.

First we construct a $P_{5}$-saturated graph of size $m=\left\lceil\frac{5 n-4}{6}\right\rceil$. Let $T_{a, b}$ be a graph obtained from two stars $S_{a}$ and $S_{b}$ by adding an edge joining the two centers (see Figure $2)$. We can construct the following graphs: $k T_{2,2}, \quad(k-1) T_{2,2} \cup T_{2,3}, \quad k T_{2,2} \cup K_{2}, \quad(k-$ 1) $T_{2,2} \cup T_{2,3} \cup K_{2}, \quad(k-1) T_{2,2} \cup T_{3,3} \cup K_{2}, \quad(k-1) T_{2,2} \cup T_{4,3} \cup K_{2}$ in line with $i$-values. For example, we can take $G=T_{2,2} \cup T_{4,3} \cup K_{2}$ if $n=17$.

Then for the values of $m \in\left(\left\lceil\frac{5 n-4}{6}\right\rceil, n\right)$, we can build a $P_{5}$-saturated $(n, m)$ graph by the following process starting with saturated graphs with $\left\lceil\frac{5 n-4}{6}\right\rceil$ edges: $T_{a, b}+T_{x, y} \Rightarrow$ $T_{x+a+1, y+b+1}$ or $T_{x, y}+K_{2} \Rightarrow T_{x+1, y+1}$. This process is shown on Figure 4.3. At the end of this process we shall get a $P_{5}$-saturated with $n-1$ edges in this type of tree $T_{a, n-a-2}$.

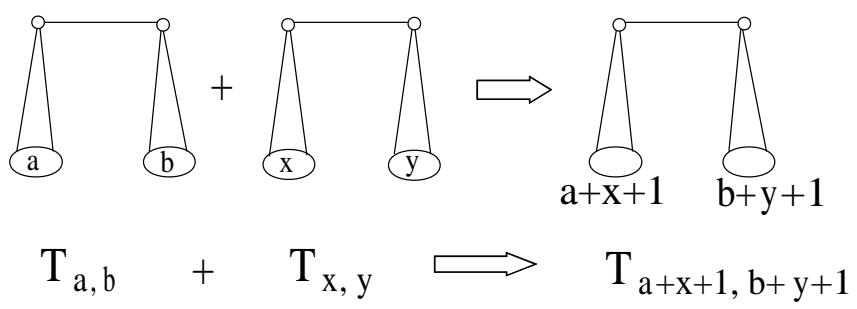

Figure 4.3: The Evolution of $P_{5}$-saturated Trees

Now we are ready to give the edge spectrum of $P_{5}$-saturated graphs and present one of the main theorems. The interval $[A, B]=\left[\operatorname{sat}\left(n, P_{5}\right)\right.$, ex $\left.\left(n, P_{5}\right)\right]$ can be obtained from Theorem 4.2.1 and Corollary 4.2.3. We want to determine whether this interval is the spectrum of $P_{5}$ or are there any missing values within this interval.

It is worth noting here that at each induction process we jump 4 steps. First we give the results on four initial values of $n$, which are listed in the table, then apply the induction process to get results on the next four $n$ values.

Theorem 4.3.6 Let $n \geq 5$ and $\operatorname{sat}\left(n, P_{5}\right) \leq m \leq e x\left(n, P_{5}\right)$ be integers. There exists an 
$(n, m) \operatorname{graph} G \in S A T\left(n, P_{5}\right)$ if and only if $n=1,2(\bmod 4)$, or

$$
m \notin \begin{cases}\left\{\frac{3 n-5}{2}\right\} & \text { if } n \equiv 3(\bmod 4) \\ \left\{\frac{3 n}{2}-3, \frac{3 n}{2}-2, \frac{3 n}{2}-1\right\} & \text { if } n \equiv 0(\bmod 4)\end{cases}
$$

Proof. The proof of this result follows from Lemma 4.3.1 through Lemma 4.3.5 and the next table, by induction on $n$.

\begin{tabular}{|c|c|c|c|c|c|}
\hline$n$ & {$[A, B]$} & $S A T\left(n, P_{5}\right)$ & $\mathrm{n}$ & {$[A, B]$} & $S A T\left(n, P_{5}\right)$ \\
\hline \multirow{3}{*}{5} & \multirow{3}{*}[4,6]{} & $4: K_{3} \cup K_{2}$ & \multirow{3}{*}{6} & \multirow{3}{*}[5,7]{} & $5: T_{2,2}$ \\
\hline & & $5: S_{4}^{+}$ & & & $6: 2 K_{3}$ \\
\hline & & $6: K_{4} \cup K_{1}$ & & & $7: K_{4} \cup K_{2}$ \\
\hline \multirow{5}{*}{7} & \multirow{5}{*}[6,9]{} & $6: T_{2,3}$ & \multirow{5}{*}{8} & \multirow{5}{*}[6,12]{} & 6: $T_{2,2} \cup K_{2}$ \\
\hline & & $7: 2 K_{3}$ & & & $7: T_{3,3}$ \\
\hline & & $8: \emptyset$ & & & 8: $K_{3} \cup S_{4}^{+}$ \\
\hline & & 9: $K_{4} \cup K_{3}$ & & & $9-11: \emptyset$ \\
\hline & & & & & 12: $2 K_{4}$ \\
\hline
\end{tabular}

In the above table, the symbol ' $\emptyset$ ' stands for no $(n, m) P_{5}$-saturated graph exists and $A=\operatorname{sat}\left(n, P_{5}\right), B=\operatorname{ex}\left(n, P_{5}\right)$. The nonexistent of $P_{5}$-saturated graphs comes from Lemma 3.3 by counting the edges.

The initial results for $n=5,6,7,8$ are listed in the above table. By the induction hypothesis suppose we have the result on $n$, then we shall apply Lemma 4.3.4 to get some result on $n+4$. Once we determined all possible $P_{5}$-saturated graph with $n$ vertices, we could also determine the $P_{5}$-saturated graph with $n+4$ vertices according to Lemma 4.3.4. Therefore we can cover the interval $\left[\operatorname{sat}\left(n, P_{5}\right)+6\right.$, ex $\left.\left(n, P_{5}\right)+6\right]$ which is exactly the interval $\left[\operatorname{sat}\left(n, P_{5}\right)+6, \operatorname{ex}\left(n+4, P_{5}\right)\right]$. In order to finish the argument that we can determine all integers between $\operatorname{sat}\left(n+4, P_{5}\right)$ and $e x\left(n+4, P_{5}\right)$, we also need to deal with the subinterval $\left[\operatorname{sat}\left(n+4, P_{5}\right)\right.$, sat $\left.\left(n, P_{5}\right)+5\right]$, which is fortunately covered by Lemma 4.3.5 since $\operatorname{sat}\left(n, P_{5}\right)+5 \leq(n+4)-1$ for any integer $n \geq 9$. 


\subsection{Edge Spectrum of $P_{6}$-Saturated Graphs}

If $G$ is a $P_{6}$-saturated graph with order less than 6 , then $G$ must be a complete graph. What's more, the order of the union of any two components of $G$ must be at least 6 . Let $B_{n}$ denote the Book graph, the union of triangles sharing one edge. A $\theta$-graph is the union of three internally disjoint (simple) paths that have the same two distinct end vertices. As we did on the case of $P_{5}$-saturated graph, we shall pay attention to each connected component of $G$.

Lemma 4.4.1 Let $H$ be a 2-connected $P_{6}$-saturated graph of order at least 6 . Then $H$ must be a book.

Proof. Let $C$ be the longest circuit in $H$. The length of $C$ must be less than 5 since otherwise $P_{6}$ would be found in subgraph obtained by $C$ and any other edge touching it. On the other hand, the length of $C$ can not be less than 4 since $H$ is 2-connected and every vertex outside of the circuit $C$ is connected to $C$ by two edge-disjoint paths. Therefore $C$ is a circuit of length 4 and denote $C=u x v y$, where $e=u v \notin E(H)$ is a missing edge in $H$ since $H$ is not complete.

Considering the fact that every edge in $H$ must have an endpoint in $C$, then the vertex set $H_{C}=H-C$ is an independent set. Take $w \in H_{C}$, then $w$ is adjacent to at least two vertices in $C$ from the fact that $H$ is 2-connected, and $w$ can not be connected to consecutive two vertices in $C$ since otherwise we can find a circuit larger than $C$. Thus $w$ is connected to either $u$ and $v$ or $x$ and $y$. Since we need to avoid the appearance of $P_{6}$, all vertex from $H_{C}$ must be connected to either $u$ and $v$ or $x$ and $y$.

If all the vertices are connected to $u$ and $v$, then $H$ is a $\theta$-graph and we can add the edge $e$ to $H$, the resulting graph $H+e$ does not contain $P_{6}$. Therefore all the other vertices are connected to $x$ and $y$. By the same argument the edge $x y$ must be in $H$, hence $H$ contains the subgraph $H_{x y}$, where $H_{x y}$ is a book with common edge $x y$. In addition, any other edge added to $H$ will result to a copy of $P_{6}$, i.e. $H$ is a book, as desired.

Lemma 4.4.2 Let $H$ be a connected $P_{6}$-saturated graph with cut vertices, then each block of $H$ must be a clique. What's more, if $H_{1}$ and $H_{2}$ are two blocks in $H$, then $\left|V\left(H_{1}\right)\right|+$ $\left|V\left(H_{2}\right)\right| \leq 6$. 
Proof. The result is trivial if $H$ is a tree. Let $v$ be the cut vertex and $H_{v}$ be one of nontrivial blocks containing $v$ in $H$. If $\left|H_{v}\right| \leq 4$, then $H_{v}$ is complete.

Hence we may assume that $\left|H_{v}\right| \geq 5$, and let $C$ be the longest circuit in block $H_{v}$ with $|C|=k$. It's easy to find a path with length at least 6 if $k \geq 5$. Let $C=u x v y$ be a circuit of length 4 . Considering the fact that $H$ is $P_{6}$-saturated and $v$ is its cut vertex, every vertices in the block $H_{v}$ but not in $C$ is connected to $u$ and $v$. In addition, the edge $e=u v$ must be in block $H_{v}$ since otherwise one may put it back, and the resulting graph is still $P_{6}$-free, contradicting the fact that $H$ is $P_{6}$-saturated. Hence the block $H_{v}$ is a book with common edge $e=u v$ and any other block containing $u$ is trivial. By the way, $H$ contains no more cut vertex since otherwise $P_{6}$ will pop up. That is to say, $H-H_{v}$ is an independent set. Now we can completely connect $v$ to this independent set and the resulting graph is still $P_{6}$-free graph, a contradiction.

According to the above lemmas, any connected $P_{6}$-saturated graph with order at least 6 is either a book or one of the following types, where $B_{n}$ is the book with $\mathrm{n}$ triangles sharing one common edge, $F_{n}$ is obtained from $K_{4}$ by gluing to the center of star $S_{n}, T_{n}$ is the union of $n$ triangles by sharing one common vertex and $T_{n}^{\prime}$ is obtained from $T_{n}$ by adding one more edge, $T_{i, j, k}$ is a rooted three-level tree.

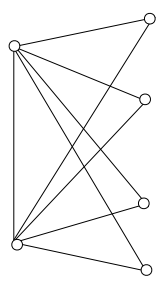

$\mathrm{B}_{4}$

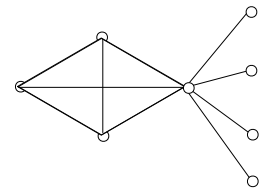

$\mathrm{F}_{4}$

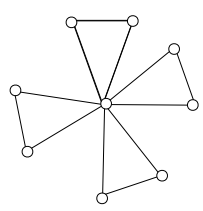

$\mathrm{T}_{4}$

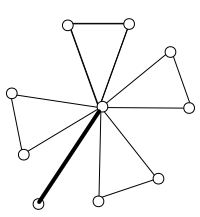

$\mathbf{T}_{4}^{\prime}$

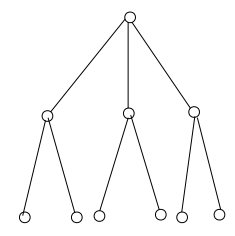

$\mathrm{T}_{2,2,2}$

Figure 4.4: All possible structures for connected $P_{6}$-saturated graphs

Let $G$ be a $P_{6}$-saturated graph. Denote by $a, b, c, \alpha, \beta_{1}, \beta_{2}, \gamma, \delta$ be the number components isomorphic to the various structures $K_{3}, K_{4}, K_{5}, F_{i}, T_{j}, T_{j^{\prime}}^{\prime}$, trees and books in $G$ for the remainder of this section. For any graph $G$, we denote by $r(G)=|E(G)|-|V(G)|$ the rank of $G$. Thus $r\left(K_{4}\right)=r\left(F_{i}\right)=2, r\left(K_{5}\right)=5$. 
Lemma 4.4.3 Let $m=2 n-4$ and $n \geq 10$ be positive integers. There exists an $(n, m)$ graph in $\operatorname{SAT}\left(n, P_{6}\right)$ if and only if $n \equiv 1,3(\bmod 5)$.

Proof. Let $n \equiv 1(\bmod 5)$ and $m=2 n-4$. We can construct an $(n, m)$ graph $G=$ $F_{2} \cup \alpha K_{5}$, where $\alpha=(n-6) / 5$. Then $|V(G)|=5 \alpha+6$ and $|E(G)|=10 \alpha+8=2 n-4$. If $n \equiv 3(\bmod 5)$, we build an $(n, m)$ graph $G=2 K_{4} \cup \alpha K_{5}$, where $\alpha=(n-8) / 5$. Then $|V(G)|=5 \alpha+8$ and $|E(G)|=10 \alpha+12=2 n-4$.

Next we shall prove by way of contradiction that there is no $(n, m)$ graph in $\operatorname{SAT}\left(n, P_{6}\right)$ with $m=2 n-4$ if $n \not \equiv 1,3(\bmod 5)$. Suppose $G$ is such a graph with components $H_{1}, H_{2}, \cdots, H_{l}$ and $h_{1} \leq h_{2} \leq \cdots \leq h_{l}$, where $h_{i}=\left|H_{i}\right|$. If $h_{1}=1$, then each component $H_{i}$ would be $K_{5}$ for every $i \neq 1$. Therefore we have $n=1(\bmod 5)$, a contradiction. If $c_{1}=2$, the remaining components of $G$ would be either a $K_{4}, K_{5}, F_{i}$ or a book $B_{j}$. Then we have

$$
n=2+4 b+5 c+\sum_{i=1}^{\alpha}\left|V\left(F_{i}\right)\right|+\sum_{j=1}^{\delta}\left|V\left(B_{j}\right)\right|
$$

and

$$
m=1+6 b+10 c+\sum_{i=1}^{\alpha}\left(\left|V\left(F_{i}\right)\right|+2\right)+\sum_{j=1}^{\delta}\left(2\left|V\left(B_{j}\right)\right|-3\right)
$$

where $\left|V\left(F_{i}\right)\right|,\left|V\left(B_{j}\right)\right| \geq 6$. Hence $4=2 n-m=3+2 b+\sum_{i=1}^{\alpha}\left(\left|V\left(F_{i}\right)\right|-2\right)+3 \delta$. Simplifying it we have

$$
2 b+\sum_{i=1}^{\alpha}\left(\left|V\left(F_{i}\right)\right|-2\right)+3 \delta=1
$$

It's easy to see that there is no integer solution to this equation.

So far we may suppose that $h_{1} \geq 3$, and the remaining components of $G$ are $K_{3}, K_{4}, K_{5}, F_{i}$, $T_{j}, T_{j^{\prime}}^{\prime}$, trees $T^{(k)}$ and books $B_{l}$. By counting the number of vertices and edges of each component, we have

$$
n=3 a+4 b+5 c+\sum_{i=1}^{\alpha}\left|V\left(F_{i}\right)\right|+\sum_{j=1}^{\beta_{1}}\left|V\left(T_{j}\right)\right|+\sum_{j^{\prime}=1}^{\beta_{2}}\left|V\left(T_{j^{\prime}}^{\prime}\right)\right|+\sum_{k=1}^{\gamma}\left|V\left(T^{(k)}\right)\right|+\sum_{l=1}^{\delta}\left|V\left(B_{l}\right)\right|
$$

and 


$$
\begin{aligned}
m=3 a+6 b+10 c & +\sum_{i=1}^{\alpha}\left(\left|V\left(F_{i}\right)\right|+2\right)+\sum_{j=1}^{\beta_{1}} \frac{3\left(\left|V\left(T_{j}\right)\right|-1\right)}{2}+\sum_{j^{\prime}=1}^{\beta_{2}}\left(\frac{3\left|V\left(T_{j^{\prime}}^{\prime}\right)\right|}{2}-2\right) \\
& +\sum_{k=1}^{\gamma}\left(\left|V\left(T^{(k)}\right)\right|-1\right)+\sum_{l=1}^{\delta}\left(2\left|V\left(B_{l}\right)\right|-3\right),
\end{aligned}
$$

where $\left|V\left(F_{i}\right)\right|,\left|V\left(T_{j}\right)\right|,\left|V\left(T_{j^{\prime}}^{\prime}\right)\right|,\left|V\left(B_{l}\right)\right| \geq 6$ and $\left|V\left(T^{(k)}\right)\right| \geq 10$. Then we have

$4=2 n-m=3 a+2 b+\sum_{i=1}^{\alpha}\left(\left|V\left(F_{i}\right)\right|-2\right)+\sum_{j=1}^{\beta_{1}} \frac{\left|V\left(T_{j}\right)\right|+3}{2}+\sum_{j^{\prime}=1}^{\beta_{2}}\left(\frac{\left.\left|V\left(T_{j^{\prime}}^{\prime}\right)\right|\right)}{2}+2\right)+\sum_{k=1}^{\gamma}\left(\left|V\left(T^{(k)}\right)\right|+1\right)+3 \delta$,

which implies that $\beta_{1}=\beta_{2}=\gamma=0$, and $\alpha \leq 1$. Plugging into the above equation we have $4=2 b+3(a+\delta)+\alpha(|V(F)|-2)$. The integer solution to this equation is either $\alpha=1,|V(F)|=6, a=b=\delta=0$ or $b=2, a=\delta=\alpha=0$. The first case implies that $G$ is $F_{2}$ with 6 vertices and 8 edges, a contradiction. For the latter case, $G$ is the union of two $K_{4}$ 's and some $K_{5}$ 's, implying that $n=8+5 c$, contradicting the assumption that $n \not \equiv 1,3(\bmod 5)$.

By a similar argument we have the following lemma.

Lemma 4.4.4 If $n$ is an integer and divisible by 5, then there is no $(n, m)$ graph in $S A T\left(n, P_{6}\right)$ with $m=2 n-2$ or $m=2 n-1$.

Proof. We prove this by way of contradiction. Suppose $G$ is such an $(n, m)$ graph in $S A T\left(n, P_{6}\right)$ with $2 n-m \in\{1,2\}$. Then $h_{1} \geq 2$. If $h_{1}=2$, then $2 n-m=3+2 b+$ $\sum_{i=1}^{\alpha}\left(\left|V\left(F_{i}\right)\right|-2\right)+3 \beta \geq 3$, a contradiction. Hence we may assume that $h_{1} \geq 3$. Arguing as we did in the previous lemma we have

$2 n-m=3 a+2 b+\sum_{i=1}^{\alpha}\left(\left|V\left(F_{i}\right)\right|-2\right)+\sum_{j=1}^{\beta_{1}} \frac{\left|V\left(T_{j}\right)\right|+3}{2}+\sum_{j^{\prime}=1}^{\beta_{2}}\left(\frac{\left.\left|V\left(T_{j^{\prime}}^{\prime}\right)\right|\right)}{2}+2\right)+\sum_{k=1}^{\gamma}\left(\left|V\left(T^{(k)}\right)\right|+1\right)+3 \delta$.

The constraint $2 n-m \in\{1,2\}$ implies that $b=1, a=\alpha=\beta_{1}=\beta_{2}=\gamma=\delta=0$ and hence $G$ is the union of $K_{4}$ and some $K_{5}$ 's. Therefore we have $n=4+5 c$, contradicting the fact that $5 \mid n$. 
Lemma 4.4.5 Let $n$ be an integer at least 15, then there is a $P_{6}$-saturated $(n, m)$ graph for all $\left\lceil\frac{9 n}{10}\right\rceil \leq m \leq n+5$.

Proof. For those values of $m$ in $[n, n+5]$, we can construct $P_{6}$-saturated graphs as : $T_{2}^{\prime} \cup T_{2,2, n-14}, \quad K_{2} \cup F_{n-6}, \quad F_{n-4}, \quad K_{2} \cup K_{4} \cup F_{n-10}, \quad K_{4} \cup F_{n-8}, \quad B_{4} \cup F_{n-10}$.

Let $n=10 k+i$, where $i=0,1, \cdots, 9$. we can construct an $(n, m)$ graph in $\operatorname{SAT}\left(n, P_{6}\right)$ as follows: $G_{0}=(k-1) T_{2,2,2} \cup T_{2,2,2+i}$, then $m=|E(G)|=9(k-1)+9+i=9 k+i=\left\lceil\frac{9 n}{10}\right\rceil$. Next we shall define an operation as follows: $T_{a, b, c}+T_{x, y, z} \Rightarrow T_{x, y, z+a+b+c+4}$. Under this operation we built a new $P_{6}$-saturated graph $T_{x, y, z+a+b+c+4}$ from a given $P_{6}$-saturated graph $T_{a, b, c} \cup T_{x, y, z}$ with one more edge. Continuing this process by starting from $G_{0}$, we can end this process with $T_{2,2, n-8}$, which has $n-1$ edges. Hence we find $P_{6}$-saturated graphs with $m$ edges for $\left\lceil\frac{9 n}{10}\right\rceil \leq m \leq n-1$.

Combining Lemma 4.4.1 through 4.4.5 we shall get the next main theorem about the edge spectrum of $P_{6}$. But this time we jump 5 steps at each induction process.

Theorem 4.4.6 Let $n \geq 10$ be an integer. Then there is a $P_{6}$-saturated $(n, m)$ graph if and only if $m$ is in the following interval:

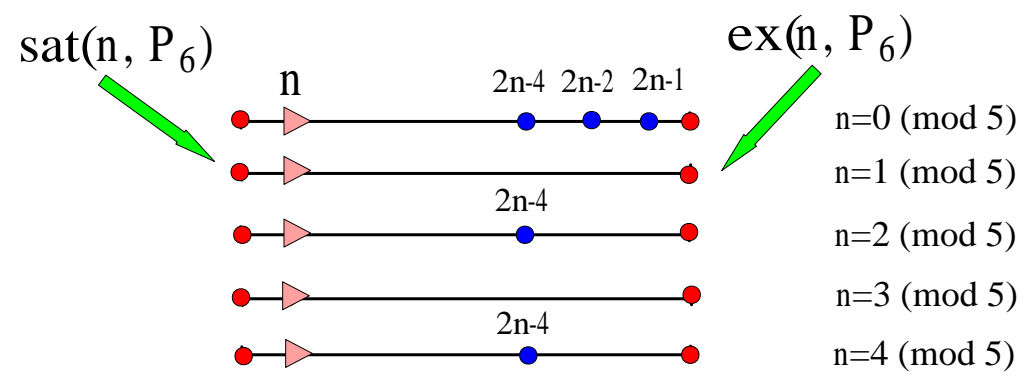

where the triangle" $\triangle "$ means the exitence of $P_{6}$-saturated $(n, n)$ graph only for $n \geq 15$, and blue dot stands for missing value.

Proof. The proof is by induction on $n$ based on the following table, where $A=\operatorname{sat}\left(n, P_{6}\right)$ and $B=\operatorname{ex}\left(n, P_{6}\right)$. The initial results for $10 \leq n \leq 14$ are listed in the table. One exception on this table is $n=11, m=14$. There is no such kind of $P_{6}$-saturated graph, but on the next induction set we do have a $(16,24)$-graph $4 K_{4}$, which is $P_{6}$-saturated 
graph. On the base case of induction process, it is easy to check the graph listed in the table. While for those not list in the table we can count all possible combinations of basic structures of $P_{6}$-saturated graph for corresponding $(n, m)$-values.

By the induction hypothesis suppose we have the result on $n \leq 14$. By adding a complete graph $K_{5}$, we may get some partial results on $n+5$. For those missing values on the interval $[A, B]$, we shall refer to Lemma 4.4.3 up to Lemma 4.4.4. So far we have covered the interval $\left[\operatorname{sat}\left(n, P_{6}\right)+10, B\right]$ since $\operatorname{ex}\left(n, P_{6}\right)+10=B$. The remaining interval $\left[A, \operatorname{sat}\left(n, P_{6}\right)+9\right]$ will be covered by Lemma 4.4 .5 since $\operatorname{sat}\left(n, P_{6}\right)+9 \leq(n+5)+5=n+10$ for any integer $n$.

\begin{tabular}{|c|c|c|c|c|c|}
\hline $\mathrm{n}$ & {$\left[\begin{array}{ll}A & B\end{array}\right]$} & $S A T\left(n, P_{6}\right)$ & $\mathrm{n}$ & {$[A, B]$} & $S A T\left(n, P_{6}\right)$ \\
\hline \multirow{11}{*}{10} & \multirow{11}{*}[9,\quad20]{} & 9: $T_{2,2,2}$ & \multirow{11}{*}{11} & \multirow{11}{*}[\begin{array}{ll}{10,}&{20}\end{array}]{} & 10: $T_{2,2,3}$ \\
\hline & & 10: $\emptyset$ & & & $11: \emptyset$ \\
\hline & & 11: $K_{2} \cup F_{4}$ & & & 12: $K_{2} \cup F_{5}$ \\
\hline & & 12: $F_{6}$ & & & 13: $F_{7}$ \\
\hline & & 13: $T_{4}^{\prime}$ & & & 14: $\emptyset$ \\
\hline & & 14: $K_{2} \cup B_{6}$ & & & $15: K_{3} \cup 2 K_{4}$ \\
\hline & & 15: $K_{4} \cup B_{4}$ & & & 16: $2 K_{3} \cup K_{5}$ \\
\hline & & 16: $\emptyset$ & & & 17: $T_{2}^{\prime} \cup K_{5}$ \\
\hline & & $17: B_{8}$ & & & 18: $K_{5} \cup F_{2}$ \\
\hline & & 18-19: $\emptyset$ & & & 19: $B_{9}$ \\
\hline & & $20: 2 K_{5}$ & & & 20: $2 K_{5} \cup K_{1}$ \\
\hline
\end{tabular}




\begin{tabular}{|c|c|c|c|c|c|}
\hline $\mathrm{n}$ & {$\left[\begin{array}{ll}A & B\end{array}\right]$} & $S A T\left(n, P_{6}\right)$ & $\mathrm{n}$ & {$[A, B]$} & $S A T\left(n, P_{6}\right)$ \\
\hline \multirow{12}{*}{12} & \multirow{12}{*}[11,21]{} & 11: $T_{2,3,3}$ & \multirow{12}{*}{13} & \multirow{12}{*}[12,23]{} & 12: $T_{3,3,3}$ \\
\hline & & $12: 4 K_{3}$ & & & $13: \emptyset$ \\
\hline & & 13: $F_{6} \cup K_{2}$ & & & 14: $K_{2} \cup F_{7}$ \\
\hline & & 14: $F_{8}$ & & & 15: $F_{9}$ \\
\hline & & 15: $F_{2} \cup K_{4} \cup K_{2}$ & & & 16: $F_{3} \cup K_{4} \cup K_{2}$ \\
\hline & & 16: $K_{4} \cup F_{4}$ & & & 17: $K_{4} \cup F_{5}$ \\
\hline & & 17: $F_{2} \cup B_{4}$ & & & 18: $T_{6}$ \\
\hline & & 18: $K_{2} \cup B_{8}$ & & & 19: $F_{2} \cup B_{5}$ \\
\hline & & 19: $K_{3} \cup K_{4} \cup K_{5}$ & & & 20: $K_{2} \cup B_{9}$ \\
\hline & & $20: \emptyset$ & & & 21: $K_{4} \cup B_{7}$ \\
\hline & & $21: 2 K_{5} \cup K_{2}$ & & & $22: 2 K_{4} \cup K_{5}$ \\
\hline & & & & & $23: K_{3} \cup 2 K_{5}$ \\
\hline \multirow{7}{*}{14} & \multirow{7}{*}[13,19]{} & "13: $T_{3,3,4}$ & \multirow{7}{*}{14} & \multirow{7}{*}[20,26]{} & 20: $F_{3} \cup B_{5}$ \\
\hline & & 14: $\emptyset$ & & & $21: F_{5} \cup K_{5}$ \\
\hline & & 15: $K_{2} \cup F_{8}$ & & & 22: $K_{2} \cup B_{10}$ \\
\hline & & 16: $F_{10}$ & & & 23: $K_{4} \cup B_{8}$ \\
\hline & & 17: $T_{2}^{\prime} \cup F_{4}$ & & & 24: $\emptyset$ \\
\hline & & 18: $K_{3} \cup T_{5}$ & & & 25: $B_{12}$ \\
\hline & & 19: $T_{6}^{\prime}$ & & & 26: $2 K_{5} \cup K_{4}$ \\
\hline
\end{tabular}




\section{Bibliography}

[1] B. Alspach, L. A. Goddyn and C.-Q. Zhang, Graphs with the circuit cover property, Trans. Amer. Math. Soc., 344(1) (1994), 131-154.

[2] K. P. Amin, The edge Spectrum of $K_{t}$-Saturated Graphs, Ph.D. Thesis, Emory University, August, 2010.

[3] Archdeacon, D. Face coloring of embedded graphs. J. Graph Theory, , 8 (1984) 387398.

[4] C. Barefoot, K. Casey, D. Fisher, K. Fraughnaugh, Size in Maximal Triangle-free Graphs and Minimal Graphs of Diameter 2, Discrete Math., 138 (1995), 93-99.

[5] J.C. Bermond, B. Jackson and F. Jaeger, Shortest coverings of graphs with cycles, J. Combin. Theory Ser. B., 35(1983), 297-308.

[6] J. A. Bondy and U. S. R. Murty, Graph Theory with Applications, Macmillan, London, (1976).

[7] Bondy, J. A. Small cycle double covers of graphs. Pages 21-40 of: Hahn, G., Sabidussi, G., and Woodrow, R. (eds), Cycles and Rays. NATO ASI Ser. C. Dordrecht: Kluwer Academic Publishers, (1990).

[8] J. A. Bondy and U. S. R. Murty, Graph Theory, Springer, (2008).

[9] Brinkmann, G., Goedgebeur, J., Hägglund, J., and Markström, K. 2011. Generation and properties of snarks. Preprint. 
[10] Celmins, U. A. On cubic graphs that do not have an edge 3-coloring, Ph.D. thesis, University of Waterloo (1984) Ontario, Canada.

[11] G. Chartand and L. Lesniak, Graphs and Digraphs, Third Edition, Chapman and Hall, 1996.

[12] R. Diestel, Graph Theory, third edition, Springer-Verlag, (2005).

[13] R. Diestel, Graph Theory, Fourth edition. Springer (2010).

[14] J. Edmonds, Maximum matching and a polyhedron with 0,1-vertices, J. Res. Nat. Bur Stand B, Math \& Math Phys. 69B (1965), pp. 125-130.

[15] P. Erdős, A. Hajnal, and J. W. Moon , A Problem in Graph Theory, Amer. Math. Monthly, 71 (1964), 1107-1110.

[16] G.-H. Fan, Covering graphs by cycles, SIAM J. Discrete Math., 5(1992), 491-496.

[17] G. Fan and A. Raspaud, Fulkerson's conjecture and circuit covers, J. Combin. Theory Ser. B., 61 (1994), 133-138.

[18] R.J. Faudree and R.H.Schelp, Path Ramsey Numbers in Multicolorings, J. Combin. Theory Ser. B., 19 (1975), 150-160.

[19] Fleischner, H. Eine gemeinsame Basis für die Theorie der eulerschen Graphen und den Satz von Petersen. Monatsh. Math., 81 (1976) 267-278.

[20] H. Fleischner, Some blood, sweat, but no tears in eulerian graph theory, Congr. Numer., 63 (1988), 9-48.

[21] H. Fleischner, Cycle decompositions, 2-coverings, removable cycles and four-color disease, Progress in Graph Theory, New York: Academic Press (1984), 233-246.

[22] H. Fleischner, Uniqueness of maximal dominating cycles in 3-regular graphs and Hamiltonian cycles in 4-regular graphs. J. Graph Theory, 18 (1994), 449459.

[23] H. Fleischner, Proof of the strong 2-cover conjecture for planar graphs, J. Combin. Theory Ser. B., 40 (1986), 229-230. 
[24] H. Fleischner and R. Häggkvist, Circuit double covers in special types of cubic graphs, Discrete Math., 309 (2009), 5724-5728.

[25] Fleischner, H., Eulerian Graph. Pages 17-53 of: Beineke, L. W., and Wilson, R. J. (eds), Selected Topics in Graph Theory (2). London: Academic Press (1983).

[26] Fleischner, H. Communication at Cycle Double Cover Conjecture Workshop, Barbados, February 25-March 4, 1990.

[27] H. Fleischner, Personal communication, at 6th Workshop on the Matthews-Sumner Conjecture and Related Problems, Domazlice, Czech Republic, April, 2011.

[28] D.R. Fulkerson, Blocking and anti-blocking pairs of polyhedra, Math. Programming, 1 (1971), 168-194.

[29] L. A. Goddyn, Cycle Covers of Graphs, Ph. D. Thesis to the University of Waterloo, 1988.

[30] L. A. Goddyn, Cones, lattices and Hilbert bases of circuits and perfect matching, Contemp. Math. AMS, 147 (1993), 419-440.

[31] L. A. Goddyn, J. van den Heuvel and S. McGuinness, Removable circuits in multigraphs, J. Combin. Theory Ser. B., 71 (1997), 130-143.

[32] L. A. Goddyn, A girth requirement for the double cycle cover conjecture, in Cycles in Graphs (B. Alspach and C. Godsil,eds.), Ann. Discrete Math., , 27 (1985) p.13-26.

[33] L. Kászonyi and Zs. Tuza, Saturated Graphs with Minimal Number of Edges, J. Graph Theory, 10:2 (1986), 203-210.

[34] G. Mazzuoccolo, The equivalence of two conjectures of Berge and Fulkerson, J. Graph Theory, 68 (2011), 125-128.

[35] G. Mazzuoccolo, Covering a 3-graph with perfect matchings, arXiv:1111.1871v2, Nov (2011).

[36] Häggkvist, R., and McGuinness, S. Double covers of cubic graphs with oddness 4, J. Combin. Theory Ser. B., , 93, (2005) 251-277. 
[37] R. Häggkvist, Lollipop Andrew strikes again, 22nd British Combinatorial Conference, July 5-10, 2009, University of St Andrews, UK.

[38] I. Holyer, The NP-completeness of edge-coloring, SIAM J. Comput., 10 (1981), 718720 .

[39] A. Huck, Reducible configurations for the cycle double cover conjecture, Discrete Applied Math., 99(1) (2000), 71-90.

[40] Huck, A., and Kochol, M. Five cycle double covers of some cubic graphs, J. Combin. Theory Ser. B., , 64, (1995) 119-125.

[41] Huck, A. POn cycle-double covers of graphs of small oddness, Discrete Math., , 229 (2001) 125-165.

[42] A. Itai and M. Rodeh, Covering a graph by circuits, Lecture Notes in Computer Science, vol. 62, Berlin: Springer-Verlag, (1978), 289-299.

[43] Jackson, B., On circuit covers, circuit decompositions and Euler tours of graphs. Pages 191-210 of: Walker, K. (ed), Surveys in Combinatorics, London Math. Soc. Lecture Note Series, vol. 187. Cambridge (1993) Cambridge University Press.

[44] F. Jaeger, Nowhere-zero Flow Problems, Pages 71-95 of : Selected Topics in Graph Theory 3, London: Academic Press.

[45] Jaeger, F. A survey of the cycle double cover conjecture Pages 1-12 of: Alspach, B., and Godsil, C. (eds), Cycles in Graphs. Ann. Discrete Math., , vol. 27 (1985) Amsterdam: North-Holland.

[46] T. Kaiser, D. Kral, and S. Norine, Unions of perfect matching in cubic graphs, Topics in discrete mathematics, Algorithms Combin., 26 (2006), 225-230, Springer, Berlin.

[47] H.-J. Lai and C.-Q. Zhang, Hamilton Weights and Petersen Minor, J. Graph Theory, 38 (2001), 197-219.

[48] G. Mazzuoccolo, The equivalence of two conjectures of Berge and Fulkerson, Journal of Graph Theory, 68 (2011), pp. 125-128. 
[49] G. Mazzuoccolo, Covering a 3-graph with perfect matchings, arXiv:1111.1871v2, Nov (2011).

[50] McGuinness, S., The double cover conjecture. Ph.D. thesis, (1984) Queen's University, Kingston, Ontario, Canada.

[51] V. Patel, Unions of perfect matchings in cubic graphs and implications of the BergeFulkerson, CDAM Research Report, LSE-CDAM-2006-06.

[52] O. Pikhurko, Results and Open Problems on Minimum Saturated Hypergraphs, Ars Combin., 72 (2004), 111-127.

[53] Preissmann, M. Sur les colorations des arêtes des graphes cubiques, Thèse de Doctorat de 3eme. Ph.D. thesis, Universitè de Grenoble (1981) France.

[54] P. Seymour, Summs of circuits, Pages 342-355 of : Graph Theory and Related Topics. New York: Academic Press.

[55] Z. Shao, X. Xu, X. Shi and L. Pan, Some Three-Color Ramsey Numbers, Europ. J. Combinatorics, 30 (2009), 396-403.

[56] G. Szekeres, Polyhedral decompositions of cubic graphs, Bull. Austral. Math. Soc. 8 (1973), 367-387.

[57] Tarsi, M.. Semi-duality and the cycle double cover conjecture, J. Combin. Theory Ser. B., 41, (1986) 332-340.

[58] A.G. Thomason, Hamilton Cycles and Uniquely 3-Edge Colorable Graphs, Ann. Discrete Math., 3 (1978), 259-268.

[59] P. Turán, Eine Extremalaufgabe aus der Graphentheorie, Mat. Fiz. Lapok, 48 (1941), 436-452.

[60] Tutte, W. T., Personal correspondence with H. Fleischner (July 22, 1987).

[61] D.B. West, Introduction to Graph Theory Upper Saddle River, NJ: Prentice Hall (1996). 
[62] C.-Q. Zhang, Cycle Covers and Cycle Decompositions of Graphs, Ann. Discrete Math., 55 (1993), 183-190.

[63] C.-Q. Zhang, Integer Flows and cycle covers of graphs, Marcel Dekker, New York, 1997.

[64] C.-Q. Zhang, Circular Flows of Nearly Eulerian Graphs and Vertex-Splitting, J. Graph Theory, 40 (2002) 147-161.

[65] C.-Q. Zhang, Cycle Covers(I) - Minimal Contra Pairs and Hamilton Weights, J. Combin. Theory Ser. B., 100 (2010), 419-438.

[66] C.-Q. Zhang, Circuit Double Cover of Graphs, Cambridge Press (2012) 